\title{
Estudio de Mortalidad Perinatal en 21 Instituciones Colombianas
}

\author{
FEDERACION COLOMBIANA DE SOCIEDADES DE OBSTETRICIA Y GINECOLOGIA \\ SOCIEDAD COLOMBIANA DE OBSTETRIĆIA Y GINECOLOGIA \\ COLCIENCIAS
}

\section{INTRODUCCION}

La Federación Colombiana de Sociedades de Obstetricia y Ginecología, conciente de su papel como entidad científica rectora de todos los procesos de investigación en el área obstétrico-perinatal, se ha propuesto llevar a cabo estudios colaborativos con el fin de conocer adecuadamente la situación de salud de los individuos que componen el grupo de población objetivo de las áreas antes mencionadas.

Es así como en el área perinatal, ha propiciado varios estudios entre los que se cuentan el de mortalidad perinatal en instituciones de Bogotá, realizado durante 1985 y el que hoy se presenta a consideración, que valora este indicador en diversas instituciones del país.

Su propósito es analizar la situación de la mortalidad perinatal en diversas instituciones del país, con el fin de generar acciones enfocadas a mejorar las condiciones de binomio maternofetal y del recién nacido. Para el logro del mismo se debe contar con la presencia participante de las entidades que realizan la normalización, y trazan las políticas de salud en el país, tanto del sector oficial, como del privado y mixto.

$\mathrm{Su}$ objetivo general es determinar en forma consistente y confiable la mortalidad perinatal a nivel institucional en diferentes regiones de Colombia. Para cumplir esta meta se plantearon objetivos específicos que tienen como fin, dar apoyo logístico para lograr una eficacia acorde con las expectativas.
Para el desarrollo de la investigación se trazó un cronograma detallado de todas las etapas del estudio, tomando como universo la totalidad de casos de muerte perinatal que ocurrieron en las instituciones participantes desde el 1o. de julio de 1986 hasta el 30 de junio de 1987.

Para la recolección de información se elaboró un formulario que tuvo como base el anterior, utilizado en la investigación de mortalidad perinatal de Bogotá en 1985.

Una vez detectado un caso, se diligenció el formulario que contemplaba antecedentes de la muerte perinatal, tanto desde el punto de vista materno como fetoneonatal; por lo anterior el diseño investigativo fue de carácter retrospectivo.

Posteriormente se llevó a cabo el análisis de la información, que arrojó valoración descriptiva de variables simples, y de cruces de variables; este análisis tuvo como base la información producida en un programa de sistemas diseñado cuidadosamente para responder al objetivo del proyecto.

El documento consta de dos partes a saber: Una primera donde se expone el marco teórico, la justificación, los antecedentes, la metodología y el desarrollo y organización del estudio. Una segunda, donde se explican con amplitud los resultados de la investigación, así como las conclusiones y recomendaciones que se plantean a los interesados en la misma.

Al final el documento tiene como anexos el formulario y su instructivo, el flujograma de la in- 
formación y la información tabulada básica de donde se tomaron los datos para el análisis.

\section{ANTECEDENTES Y JUSTIFICACION}

Si se parte de la premisa que la mortalidad perinatal es uno de los indicadores más sensibles de la calidad del proceso reproductivo (34), se comprende la necesidad de investigar este indicador en cualquier país que quiera evaluar este proceso.

Las cifras de mortalidad perinatal obtenidas de nuestra revisión bibliográfica (Tablas 1 y 2), demuestran que la tasa de mortalidad perinatal es mayor, cuanto menor es el grado de desarrollo de los países, y como en un mismo país o región, se logra una reducción de este indicador a través de los años, como resultado de intervenciones que procuran la mejoría de la salud perinatal, $(2,3,18,25,31,34)$.

Nuestras tasas actuales de mortalidad perinatal se dieron en los países desarrollados de Europa en el año de 1966 (Tabla 2) (23); veamos algunos ejemplos de esta situación: Austria tenía en 1966 , una tasa de mortalidad perinatal de $29.8 \times 1.000$
(Tabla 2) y la Secretaría de Salud de Bogotá entre 1983 y 1984 una tasa de 29.7 x 1.000 (Tabla 1). Suecia tenía en 1966, una tasa de mortalidad perinatal de 18.9 × 1.000 (Tabla 2) y el estudio interinstitucional de Bogotá en 1985 una tasa de 18.09 x 1.000 (Tabla 1).

En América Latina en los últimos 20 años se ha logrado una reducción importante de la mortalidad infantil en casi todos los países, pero esa reducción se ha obtenido a expensas de la mortalidad post-neonatal. Se estima que casi las dos terceras partes de las muertes ocurridas durante el primer año de vida, se presentan en los primeros 28 días (período neonatal); siendo esta la razón por la cual la mortalidad perinatal (fetal tardía y neonatal pre$\mathrm{COz}$ ) se convierte en un problema prioritario en los países en vías de desarrollo, haciéndose necesaria la búsqueda de estrategias que modifiquen la situación planteada, una de las cuales es lograr que la regionalización de la atención perinatal sea real y efectiva, tratando de garantizar que cada nivel de atención cuente con las condiciones de eficiencia mínimas, que permitan la atención adecuada de la gestante y el recién nacido. De igual manera es importante instaurar un proceso normativo, basado en el enfoque

TABLA 1

TASAS DE MORTALIDAD PERINATAL SEGUN DIVERSOS AUTORES

\begin{tabular}{|c|c|c|c|c|}
\hline REF. & INVESTIGADOR & ORIGEN & AÑO & TASA $\times 1.000$ \\
\hline 20 & Ochoa G. & Hospital San Vicente de Paúl-Medellín & 57 & 100.00 \\
\hline 9 & Gaviria B. & Hospital San Vicente de Paúl-Medellín & $57-59$ & 75.40 \\
\hline 21 & Ochoa G. & Hospital San Vicente de Paúl - Medellín & 74 & 48.00 \\
\hline 29 & Rodríguez $A$. & Instituto Materno Infantil - Bogotá & $74-75$ & 43.60 \\
\hline 1 & Abudu 0 . & Lagos University & $72-82$ & 42.50 \\
\hline 6 & Díaz R. & Secretaría Salud - Bogotá & $83-84$ & 29.70 \\
\hline 18 & López G. - Riaño G. & Parto Hospitalario - En Colombia & 79 & 28.90 \\
\hline 33 & Terrin $\mathrm{M}$. & Ontario - Canadá - (Fumadoras) & $60-61$ & 28.20 \\
\hline 12 & Jubiz A. - Londoño J. & Hospital San Vicente de Paúl - Medellín & 82 & 27.40 \\
\hline 15 & Langer A. & Imper México & 83 & 26.20 \\
\hline 26 & Páez R. - Acero U. & Hospital Militar Central - Bogotá & $63-73$ & 22.40 \\
\hline 33 & Terrin M. & Ontario - Canadá (No fumadoras) & $60-61$ & 22.00 \\
\hline 14 & Knutzen V. & New York U.S.A. & $75-78$ & 20.00 \\
\hline 34 & Uriza-Barragán & Bogotá - Colombia & 85 & 18.09 \\
\hline 27 & Pearson $\mathrm{J}$ & Indiana University - U.S.A. & 82 & 18.00 \\
\hline 22 & O. Driscoll & Dublín & 80 & 16.80 \\
\hline 4 & Boylan P. & Finlandia & 83 & 16.00 \\
\hline 8 & Erkkola & Finlandia & $70-75$ & 15.70 \\
\hline 16 & Lee $\mathrm{K}$. & Finlandia & 76 & 10.00 \\
\hline 8 & Erkkola & Finlandia & $76-78$ & 8.90 \\
\hline
\end{tabular}

FUENTE: Mortalidad Perinatal, (Revisión bibliográfica). 
TABLA 2

MORTALIDAD PERINATAL EN ALGUNOS PAISES DE EUROPA EN 1966

REF.: 23. OMS. - Cuadernos de Salud Pública No. 42 Ginebra 1972 (Modificado)

\section{PAIS}

\section{Suecia}

2. Bulgaria

3. Finlandia

4. Noruega

5. Suiza

6. Dinamarca

7. Rumania

8. Países Bajos

9. Checoslovaquia

10. Luxemburgo

11. Polonia

12. Alemania Oriental

13. Inglaterra y País de Gales

14. Bélgica

15. Yugoslavia

16. Francia

17. República Federal de Alemania

18. Irlanda

19. Austria

20. Escocia

21. Grecia

22. Irlanda del Norte

23. Malta

24. Hungría

25. Italia

26. Portugal
TASA $\times 1.000$

18.9

19.0

20.8

21.1

21.7

21.8

21.9

22.7

22.8

23.3

25.1

25.5

26.7

27.3

27.4

27.7

27.9

29.1

29.8

29.8

30.4

31.3

32.4

35.3

36.8

43.6 de riesgos y en esta forma lograr que los pacientes de bajo riesgo sean atendidos en los niveles I y II y los de alto riesgo en el nivel III $(3,25)$.

Se han identificado condiciones que están relacionadas con una mayor tasa de mortalidad perinatal.

1o.) El bajo peso al nacer $(1,9,12,15,29,34)$, siendo el trabajo de parto pretérmino, el que más contribuye al nacimiento de recién nacidos con pesos menores de 2.500 gramos (4, $12,15,30,34)$. Según la literatura médica, los problemas obstétricos que con más frecuencia se asocian al trabajo de parto pretérmino son: La ruptura prematura de membranas $(5,12$,
$20,30)$ y la infección urinaria o bacteriuria asintomática $(13,17)$.

2o.) Afecciones maternas como la preeclampsia y el abruptio $(1,19)$, y el abruptio, que tienen como principal factor asociado los fenómenos hipertensivos del embarazo $(19,34)$.

La identificación etiológica de la mortalidad perinatal en nuestro medio es muy difícil, especialmente en la muerte anteparto donde casi siempre la muerte fetal se clasifica como desconocida (34). La identificación etiológica de la muerte perinatal durante el parto y durante el período neonatal precoz, aun cuando es menos difícil, se ve entorpecida por la falta de autopsia del mortinato, o del que muere en el período neonatal precoz. En los casos de muerte neonatal precoz ocurridos en las unidades de cuidado intensivo neonatal, definir la causa etiológica de la muerte tal vez sea más fácil que en los otros casos y posiblemente la primera causa sea la membrana hialina (34). Teniendo en cuenta que el conocimiento de las causas que condicionan la mortalidad perinatal en nuestro medio, es de fundamental importancia para la formulación de estrategias tendientes a reducir la magnitud del problema, se hace necesario el uso de instrumentos que permitan obtener la mayor información posible y la adopción de modelos de clasificación de la causa básica de la defunción.

El estudio de mortalidad perinatal de Bogotá nos permitió concluir que el formulario de registro debía ser modificado y simplificado para su uso a nivel nacional, creando conciencia en los integrantes del equipo de salud comprometido en la a tención del grupo obstétrico perinatal, del real y verdadero valor que el indicador mortalidad perinatal tiene en la evaluación tanto del estado de salud de la población general, como de la calidad de la atención brindada.

Por todo lo anterior la Federación Colombiana de Sociedades de Obstetricia y Ginecología (Fecolsog), en su asamblea nacional realizada durante el XVI Congreso Colombiano de la especialidad que se efectuó en Cali en diciembre de 1985, decidió ampliar la investigación que sobre mortalidad perinatal se realizó en 11 Instituciones de Bogotá y cuyos resultados se presentaron como ponencia oficial en dicho Congreso, a otras Instituciones en diferentes regiones del país. La coordinación de la investigación fue asignada a la Sociedad Colombiana de Obstetricia y Ginecología (SCOG), entidad que se encargaría de nombrar el coordinador o director del proyecto. 


\section{PROPOSITOS Y OBJETIVOS}

\subsection{Propósito}

Analizar la situación de la mortalidad perinatal en las instituciones participantes, para presentar los resultados ante los organismos nacionales e internacionales responsables de la atención materno infantil, con el fin de generar acciones tendientes a mejorar las condiciones de este grupo en Colombia.

\subsection{Objetivo general}

Determinar en forma consistente y confiable la mortalidad perinatal a nivel institucional en diferentes regiones de Colombia.

\subsection{Objetivos específicos}

3.3.1. Identificar la magnitud del problema en las instituciones participantes.

3.3.2. Identificar las causas más importantes de mortalidad perinatal en las instituciones participantes.

3.3.3. Identificar algunas características de la población blanco del estudio.

3.3.4. Analizar algunos factores biológicos y bio-sociales que afectan la mortalidad perinatal.

\section{MATERIAL Y METODOS}

\subsection{Población}

Se tomó la totalidad de los casos de muerte perinatal que ocurrieron en las instituciones participantes desde el 1o. de julio de 1986, hasta el 30 de junio de 1987. Para ello se tomó el parto institucional, excluyendo el ocurrido fuera del hospital o el recién nacido remitido y que falleció en la institución.

\subsection{Recolección de la información}

Se elaboró un formulario modificando el utilizado en la investigación realizada en Bogotá en 1985 sobre registro de defunción perinatal (anexos 1 y $1 \mathrm{~A})$, con su correspondiente instructivo (Anexo 2). Se diligenció un formulario para cada caso de muerte perinatal, se contabilizaron todos los nacimientos (vivos y muertos), con el fin de calcular la tasa de mortalidad perinatal. El proceso de la recolección se puede apreciar en el flujograma (Anexo 3).

\subsection{Procesamiento de la información}

Los datos recolectados en los formularios durante el período comprendido entre julio 1o. de 1986 y junio 30 de 1987 fueron procesados por computador bajo la dirección del Ingeniero de Sistemas señor Alvaro López.

\subsection{Análisis}

Los datos procesados fueron analizados por un grupo de investigadores, en una reunión de trabajo que se realizó en el Hotel Colonial de Paipa, del 1o. al 4 de octubre de 1987; allí se elaboró el primer borrador del documento.

El grupo de análisis estuvo conformado por las siguientes personas:
Jairo Barragán

Jairo de la Cruz Segura

Martha Jiménez

Alfonso Jubiz Hasbún

Germán León

Iván Ortiz

Claudio Pérez

Arturo Rodríguez Soto

Gloria Ruiz

Germán Uriza G.
Guillermo González
Ginecólogo Obstetra

Ginecólogo Obstetra

Ginecólogo Obstetra

Patóloga

Ginecólogo Obstetra

Estadístico

Ginecólogo Obstetra

Pediatra Neonatólogo

Ginecólogo Obstetra

Ginecólogo Obstetra
Pediatra Neonatóloga

La reunión final de revisión del documento, se realizó en la sede de Ascofame el viernes 30 de octubre de 1987 con la asistencia de los Doctores: Jairo Barragán, Jairo de la Cruz S., Guillermo González, Alfonso Jubiz H., Germán León, Edgar Iván Ortiz, Arturo Rodríguez Soto y Germán Uriza G.

\section{ORGANIZACION Y DESARROLLO DE LA INVESTIGACION}

Terminado el XVI Congreso de la especialidad en Cali y adjudicada la investigación a la Sociedad Colombiana de Obstetricia y Ginecología, se enviaron cartas a todos los presidentes de las Sociedades filiales de Fecolsog recibiendo respuesta afirmativa de 10 Sociedades quienes nombraron 22 delegados que representaban a 25 instituciones de Colombia que se reunieron en la sede de Ascofame en abril 25 y 26 de 1986, donde se elaboraron el borrador del formalario con su instructivo y el protocolo de la investigación. El sábado 31 de mayo de 1986 en reunión que se llevó a cabo en Ascofame se hizo la revisión final del formularıo instructivo y protoco- 
lo; se nombraron los coordinadores institucionales, regionales y los miembros del comité central.

La recolección de la información, el procesamiento de datos y el análisis se realizó en la forma descrita en los numerales 4.2., 4.3., y 4.4.

El protocolo fue presentado a COLCIENCIAS, entidad que aprobó el estudio firmándose un convenio entre la Sociedad Colombiana de Obstetricia y Ginecología y COLCIENCIAS en noviembre 14 de 1986.

\subsection{Instituciones}

De las 25 instituciones que iniciaron la investigación, se retiraron por diversos motivos 4 a saber: Hospital Universitario de Cartagena, Rosario Pumarejo de López de Valledupar, Seguro Social de Cali, y Hospital San Pedro de Pasto. Las 21 instituciones participantes se agruparon por niveles en la siguiente forma:

\begin{tabular}{|lcc|}
\hline TIPO & NUMERO & PORCENTAJE \\
\hline Nivel primario & 1 & 4.80 \\
Nivel secundario & 4 & 19.00 \\
Nivel terciario & 16 & 76.20 \\
\hline Total & 21 & 100.00 \\
\hline
\end{tabular}

Las 25 instituciones que iniciaron la investigación se organizaron de acuerdo con su localización geográfica en 4 zonas con un coordinador en cada una de ellas.

1. ZONA DI: BOGOTA (12 Instituciones)

\begin{tabular}{|c|c|c|c|}
\hline CODIGO & NOMBRE & COORDINADOR & NIVEL \\
\hline 01 & Hospital Materno Infantil & Dr. Arturo Rodríguez S. & III \\
\hline 02 & Hospital San José & Dr. Alfonso Correa & III \\
\hline 03 & Hospital Infantil & Dr. Segundo Angel M. & III \\
\hline 04 & Iospital Militar Central & Dr. Jairo de la Cruz S. & III \\
\hline 05 & Hospital de la Samaritana & Dr. Luis E. Pontón & III \\
\hline 06 & Hospital San Ignacio & $\begin{array}{l}\text { Dr. Jairo Barragán } \\
\text { Dr. Francisco Revollo }\end{array}$ & III \\
\hline 07 & Clínica Palermo & Dr. Saúl Santoyo & III \\
\hline 08 & Fundación Santa Fe & Dr. Germán Suárez & III \\
\hline 09 & Clínica David Restrepo & Dra. Gloria Vargas & III \\
\hline & & Dr. Carlos Alviar & \\
\hline 10 & Hospital Simón Bolivar & Dr. Alfonso López & III \\
\hline 11 & Hospital San Blas & Dr. Guillermo González & II \\
\hline 12 & Hospital La Granja & Dr. Ernesto Walteros & I \\
\hline
\end{tabular}

Coordinador: Dr. Jairo Barragán

II. ZONA NORTE

\begin{tabular}{|c|c|c|c|c|}
\hline CODIGO & CIUDAD & INSTITUCIONES & COORDINADOR & NIVEL \\
\hline 13 & Cartagena & Hospital Universitario & Antonio Soto Yancés & III \\
\hline 14 & Cartagena & Hospital de Maternidad & & \\
\hline \multirow{3}{*}{15} & \multirow{3}{*}{ Valledupar } & Rafael Calvo & Antonio Soto Yancés & II \\
\hline & & Hospital Rosario & & \\
\hline & & Pumarejo de López & Armando René Garcíà & II \\
\hline TOTAL & & TRES INSTITUCIONES & & \\
\hline
\end{tabular}

Coordinador: Dr. Antonio Soto Yancés 
III. ZONA CENTRO

\begin{tabular}{|clll|}
\hline CODIGO & CIUDAD & INSTITUCIONES & COORDINADOR \\
\hline 16 & Medellín & Hospital San Vicente de Paúl & Alfonso Jubiz H. \\
17 & Cúcuta & Hospital San Juan de Dios & Manuel J. Palau \\
18 & Bucaramanga & Hospital Ramón González Valencia & Oscar Gómez León \\
\hline TOTAL & & & III \\
\hline
\end{tabular}

Coordinador: Dr. Alfonso Jubiz Hasbún

IV. ZONA SUR OCCIDENTAL

\begin{tabular}{|cllll|}
\hline CODIGO & CIUDAD & \multicolumn{1}{c|}{ INSTITUCIONES } & COORDINADOR \\
\hline 19 & Cali & Hospital Universitario del Valle & Iván Ortiz \\
20 & Cali & Instituto de Seguro Social & Iván Ortiz \\
21 & Pereira & Hospital Universitario San Jorge & Emilio Muñoz D. \\
22 & Pasto & Hospital Departamental de & III & III \\
& Paviñor Muñoz P. & Javier Muñoz P. \\
23 & Pasto & Hospital San Pedro & Carlos Olaya \\
24 & Ibagué & Hospital Federico Lleras Acosta & II \\
25 & Manizales & Hospital Universitario de Caldas & III \\
\hline TOTAL & & SIETE INSTITUCIONES & III & \\
\hline
\end{tabular}

Coordinador: Dr. Edgar Iván Ortiz

\subsection{Investigadores}

5.2.1. Coordinador General o Director del Estudio.

Dr. Germán Uriza Gutiérrez.

\subsubsection{Coordinadores Regionales:}

Zona Bogotá: Dr. Jairo Barragán

Zona Norte: Dr. Antonio Soto Yancés

Zona Centro: Dr. Alfonso Jubiz H.

Zona Sur Occidental: Dr. Iván Ortiz

\subsubsection{Comité Central:}

Dres.: Germán Uriza, Arturo Rodríguez, Jairo Barragán, Jairo de la Cruz Segura, Guillermo González.

5.3. Tipo de estudio: Retrospectivo con un análisis descriptivo.

5.4. Comités de mortalidad: Estuvo conformado, por el coordinador institucional, un representante del Departamento de Obstetricia, un neonatólogo o pediátra y en algunos casos el patólogo, la enfermera profesional y estudiantes de post-grado.

\subsubsection{Comité regional:}

Estuvo conformado por los coordinadores institucionales y el coordinador regional.

\subsubsection{Comité central:}

Estuvo conformado por: El Director del estudio, con la asesoría de cuatro coordinadores institucionales de Bogotá que fueron escogidos durante la reunión preparatoria de mayo 31 de 1986.

\subsubsection{Supervisión y asesoría:}

La supervisión y asesoría tuvo un carácter permanente dirigida a:

- Informe periódico a los niveles superiores.

Mantenimiento de la motivación del personal que participó en el estudio.

- Resolución y toma de decisiones sobre dudas en el diligenciamiento del formulario o la inclusión o exclusión de casos particulares.

- Recolección de la información: De acuerdo con el flujograma (Anexo 3). 


\section{DE FINICIONES}

Se utilizaron las definiciones y términos recomendados por el Centro Latinoamericano de Perinatología y Desarrollo Humano (CLAP), Gráfico 1 $(30,31)$.

\subsection{Mortalidad Perinatal}

Toda muerte del producto ocurrida a partir de la semana 28 de gestación y antes del séptimo día de nacido (168 horas). En caso de no conocimiento de la edad gestacional se utiliza como parámetro todo producto muerto de 1.000 gramos de peso o más.

\subsection{Mortalidad fetal}

Toda muerte del producto desde la iniciación del embarazo hasta el nacimiento.

\subsection{Mortalidad fetal tardía}

Toda muerte del producto entre las 28 semanas de gestación y el parto.

\subsection{Mortalidad neonatal precoz}

Es la muerte de un recién nacido vivo hasta antes del 7o. día de nacido (hasta 168 horas completas post-natales).

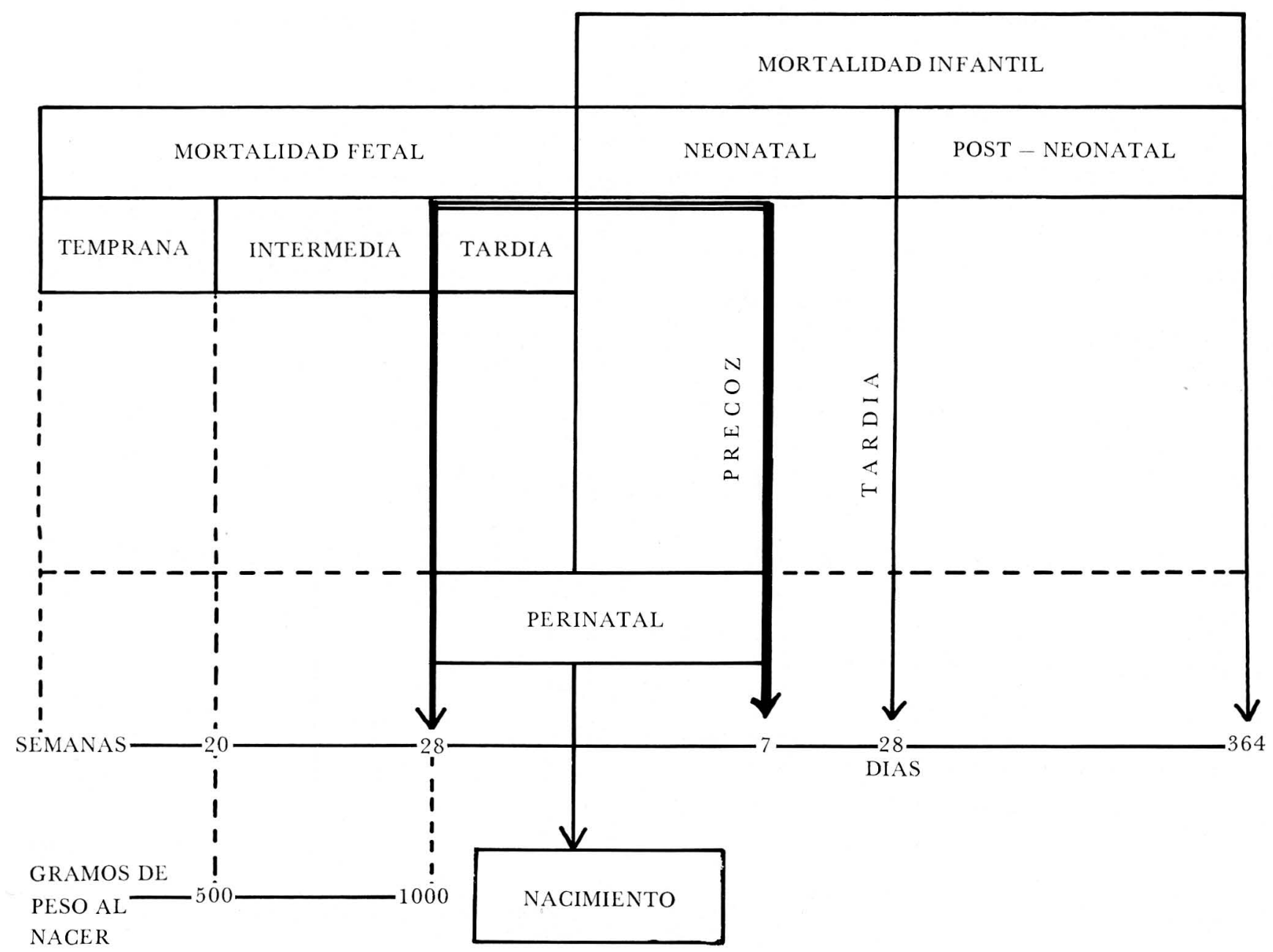




\subsection{Mortinato}

Todo niño que después del parto no presenta ninguna evidencia de vida a saber: ni respiración, ni latidos cardíacos, ni movimientos de los músculos voluntarios. Se subdividen estos mortinatos en muertes anteparto cuando el feto muere en el útero antes de iniciarse el trabajo de parto y muertes intraparto cuando la muerte ocurrió después de iniciado el trabajo de parto y antes del nacimiento (7).

\section{RESULTADOS}

Se analizaron 1.998 casos que corresponden a los formularios enviados por las 21 instituciones participantes, los cuales fueron diligenciados desde el 1o. de julio de 1986 hasta el 30 de junio de 1987 .

Para calcular la tasa de mortalidad perinatal durante el período de estudio, se tomaron todas las muertes ocurridas en las instituciones participantes que fueron 2.174, dando una diferencia en relación con los formularios recolectados de 176 , que corresponden a historias de muertes perinatales que no fueron localizadas en las instituciones de origen.

Las principales limitantes que se encontraron a través de la recolección de la información y el análisis de los resultados fueron las siguientes:

- Control y supervisión deficientes en el diligenciamiento de los formularios en algunas instituciones.

- El comité de mortalidad perinatal no funcionó en todas las instituciones.

- Sólo se realizó un número reducido de autopsias.

- Información incompleta en algunas historias clínicas institucionales.

- Problemas internos en las instituciones que interrumpieron temporalmente en algunos sitios la recolección de datos.

- Gran dificultad en el seguimiento del componente neonatal precoz de la mortalidad perinatal.

\subsection{Tasas de mortalidad perinatal según instituciones}

La mayor tasa de mortalidad perinatal se encuentra en dos instituciones de Bogotá, el Hospital
Simón Bolívar con 44.69 x 1.000 y el Hospital Materno Infantil con 41.97 x 1.000 , seguidos por el Hospital San Jorge de Pereira, con 40.24 x 1.000 . La menor tasa de mortalidad perinatal se encuentra en el único hospital de primer nivel Hospital Local La Granja, con $1.75 \times 1.000$, le sigue en orden ascendente el Hospital Infantil de Bogotá, (tercer nivel) con 8.85 x 1.000 y el Hospital San Blas (segundo nivel) con $10.94 \times 1.000$ (Tabla 3).

La tasa de mortalidad perinatal global fue $23.80 \times 1.000$; 8 instituciones tienen tasas superiores a esta tasa global. Eliminando las 64 anomalías congénitas incompatibles con la vida, que se encontraron en el estudio, la tasa corregida es de $21.17 \mathrm{x}$ 1.000 (Tabla 3).

\subsection{Tasas de mortalidad comparativa entre instituciones de Bogotá y el resto del país}

Se encontró una tasa más baja de mortalidad perinatal en Bogotá que en el resto del país (Gráfica 2), a pesar de que las dos instituciones con más alta tasa se encuentran en Bogotá (Tabla 3).

\section{GRAFICA 2}

TASAS DE MORTALIDAD PERINATAL COMPARATIVA ENTRE INSTITUCIONES DE BOGOTA Y EL RESTO DEL PAIS

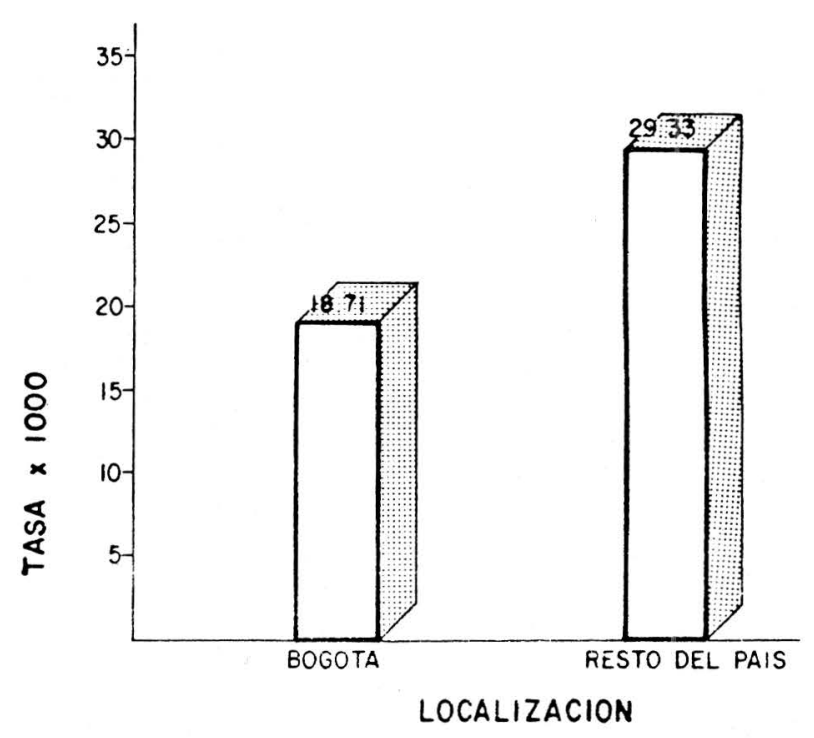

\subsection{Tasas de mortalidad perinatal por meses}

En general la tendencia durante el período de estudio fue similar, pero existe apreciable diferen- 
TABLA 3

TASAS DE MORTALIDAD PERINATAL SEGUN INSTITUCIONES

\begin{tabular}{|c|c|c|c|c|c|}
\hline ORDEN & $\begin{array}{l}\text { INSTITUCION } \\
\text { ! }\end{array}$ & NIVEL & $\begin{array}{l}(1) \\
\text { MUERTES PE- } \\
\text { RINATALES }\end{array}$ & $\begin{array}{l}\quad(2) \\
\text { NACIDOS VIVOS } \\
\text { MAS MUERTES } \\
\text { FETALES TAR- } \\
\text { DIAS. }\end{array}$ & $\begin{array}{l}\text { TASA } \\
(1) /(2) X \\
1.000\end{array}$ \\
\hline 01 & H. Simón Bolívar (Bogotá) & III & 109 & 2.439 & 44.69 \\
\hline 02 & H. Materno Infantil (Bogotá) & III & 387 & 9.219 & 41.97 \\
\hline 03 & H. San Jorge (Pereira) & III & 201 & 4.995 & 40.24 \\
\hline 04 & H. U. de Caldas (Manizales) & III & 125 & 3.649 & 34.25 \\
\hline 05 & H. U. del Valle (Cali) & III & 279 & 8.179 & 34.11 \\
\hline 06 & $\begin{array}{l}\text { lundación H. San Vicente de } \\
\text { Paúl (Medellín) }\end{array}$ & III & 99 & 2.918 & 33.92 \\
\hline 07 & $\begin{array}{l}\text { H. Ramón González Valencia } \\
\text { (Bucaramanga) }\end{array}$ & III & 148 & 4.597 & 32.19 \\
\hline 08 & H. Militar Central (Bogotá) & III & 34 & 1.403 & 24.23 \\
\hline 09 & $\begin{array}{l}\text { H. Federico Lleras Acosta } \\
\text { (Ibagué) }\end{array}$ & III & 62 & 2.804 & 22.11 \\
\hline 10 & H. Dptal. de Nariño (Pasto) & II & 56 & 2.654 & 21.10 \\
\hline 11 & $\begin{array}{l}\text { Clínica de Maternidad Rafael } \\
\text { Calvo (Cartagena) }\end{array}$ & II & 204 & 9.826 & 20.76 \\
\hline 12 & H. La Samaritana (Bogotá) & III & 30 & 1.730 & 17.34 \\
\hline 13 & H. San Ignacio (Bogotá) & III & 55 & 3.691 & 14.90 \\
\hline 14 & $\begin{array}{l}\text { Clínica de Maternidad David } \\
\text { Restrepo (Bogotá) }\end{array}$ & III & 53 & 3.579 & 14.80 \\
\hline 15 & H. San Juan de Dios (Cúcuta) & II & 110 & 7.532 & 14.60 \\
\hline 16 & $\begin{array}{l}\text { Centro Médico de los Andes } \\
\text { (Bogotá) }\end{array}$ & III & 12 & 829 & 14.47 \\
\hline 17 & H. San José (Bogotá) & III & 53 & 3.894 & 13.61 \\
\hline 18 & Clínica Palermo (Bogotá) & III & 46 & 3.824 & 12.02 \\
\hline 19 & H. San Blas (Bogotá) & II & 37 & 3.379 & 10.94 \\
\hline 20 & $\begin{array}{l}\text { H. Infantil Lorencita Villegas } \\
\text { de Santos (Bogotá) }\end{array}$ & III & 70 & 7.906 & 8.85 \\
\hline 21 & H. Local La Granja (Bogotá) & I & 4 & 2.283 & 1.75 \\
\hline & TOTAL & & 2.174 & 91.330 & 23.80 \\
\hline
\end{tabular}

cia de la tasa de mortalidad perinatal de los meses de noviembre $(27.9 \times 1.000)$ y diciembre $(20.3 \mathrm{x}$ i.000), con la tendencia general (Gráfica 3).

\subsection{Tasas de mortalidad perinatal según niveles de atención}

La tasa de mortalidad es mucho mayor en los hospitales de tercer nivel con $26.85 \times 1.000$, que en los de segundo nivel con 17.4 x 1.000 (Gráfica 4).
7.5. Distribución de las muertes perinatales según estado civil de la madre

Se encontró la gran mayoría en unión estable con $83.02 \%$, entendiendo por ésto cuando la mujer tiene un compañero permanente (Gráfica 5).

\subsection{Edad de la madre}

El promedio de la madre fue de 26 años. Se encuentran un número apreciable de casos, en los dos extremos de la vida reproductora, 127 en ado- 
GRAFICA 3

TASAS DE MORTALIDAD PERINATAL POR MESES

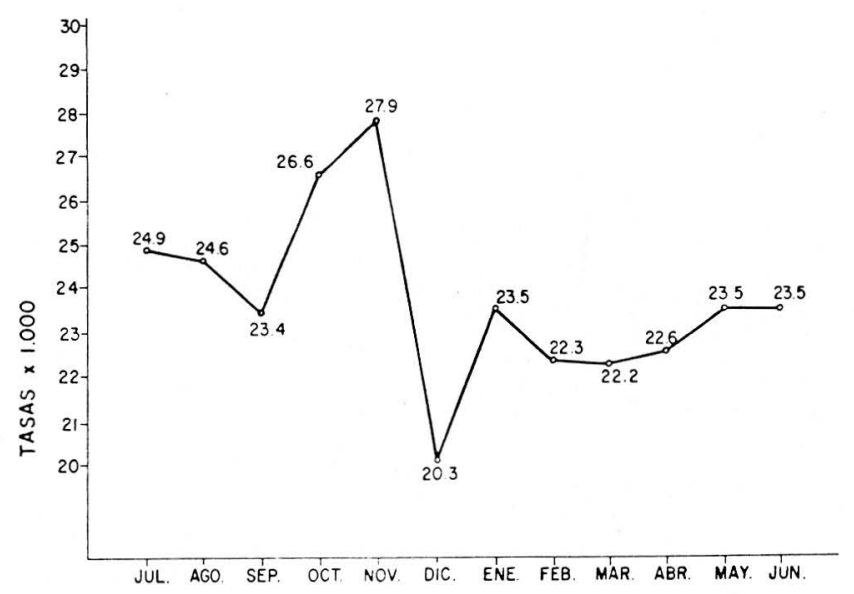

GRAFICA 4

TASA DE MORTALIDAD PERINATAL SEGUN NIVELES DE ATENCION

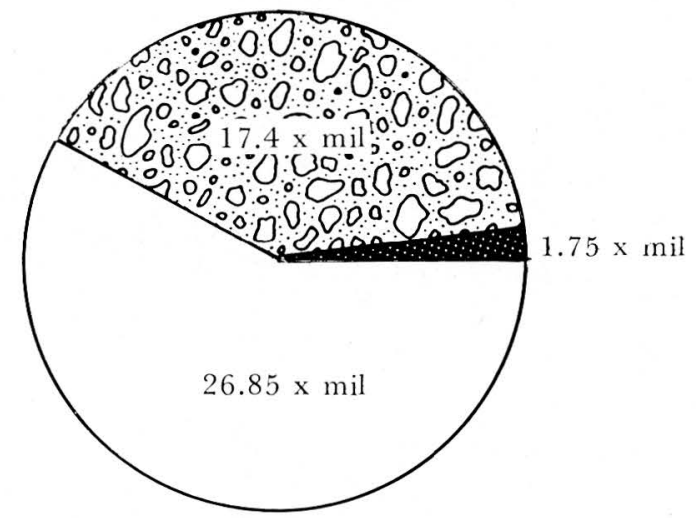

NIVEL I Od NIVEL II $\square$ NiVEL III

GRAFICA 5

ESTADO CIVIL DE LA MADRE

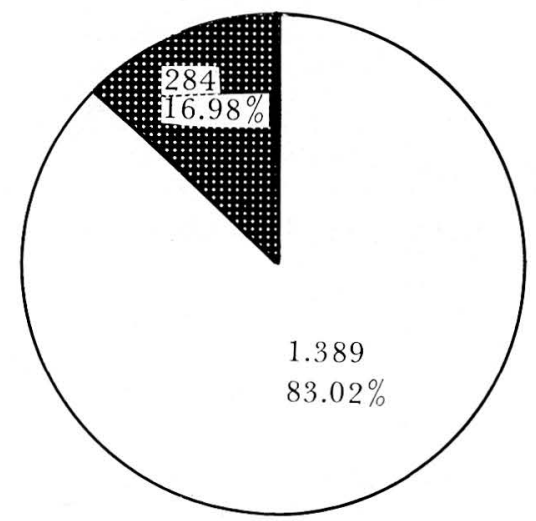

MADRE SOLA

UNION ESTABLE

SOBRE 1.673 CASOS INFORMADOS/325 SIN DATO lescentes $(6.38 \%$ ) y 121 en mayores de 37 años (6.07\%). Se encontraron 3 casos en niñas de 10 años y uno en una mujer de 50 años (Gráfica No. 6).
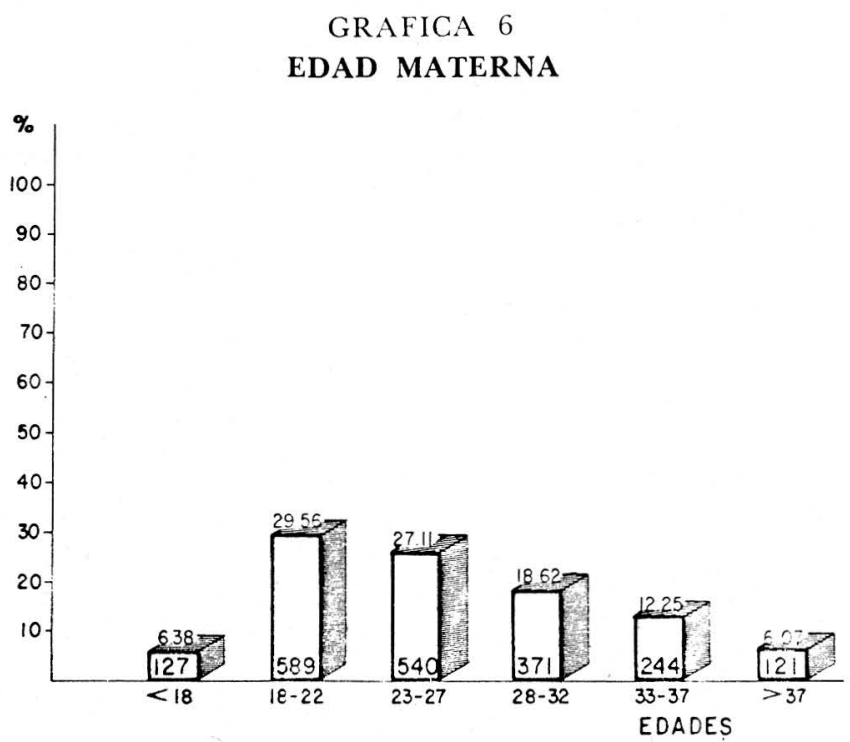

SOBRE 1992 CASOS INFORMADOS - 6 SIN DATO

En el grupo de adolescentes, más de la mitad, $54.33 \%$, son de 17 años, y 17 casos son menores de 15 años (13.39\%), distribuidos en tres pacientes de 10, 11 y 12 años; dos de 13 años y seis de 14 años. (Gráfica 7).

\section{GRAFICA 7 \\ ADOLESCENTES}

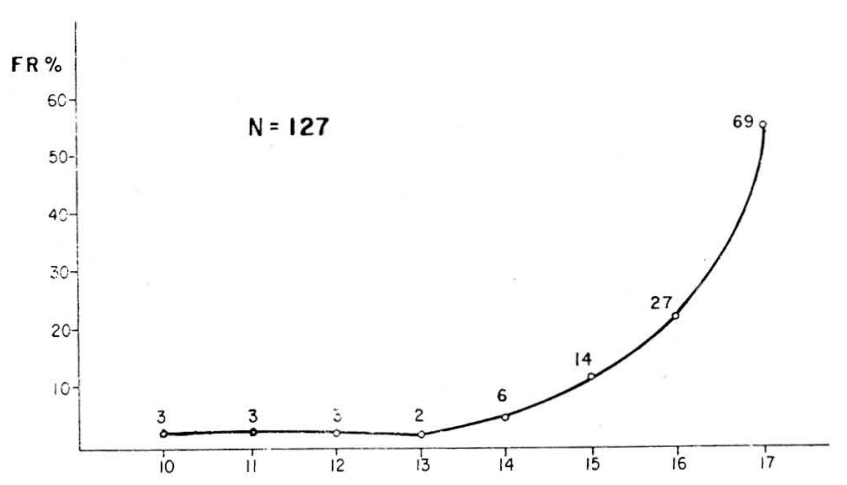

7.7. Distribución de las muertes perinatales según número de embarazos previos

Dos de cada tres pacientes tenían una o más gestaciones previas $(69.5 \%)$ de las cuales tenían entre 1 y 4 partos, el $56.48 \%$, y 5 más el $13.02 \%$. (Gráfica 8). 
GRAFICA 8

EMBARAZOS PREVIOS

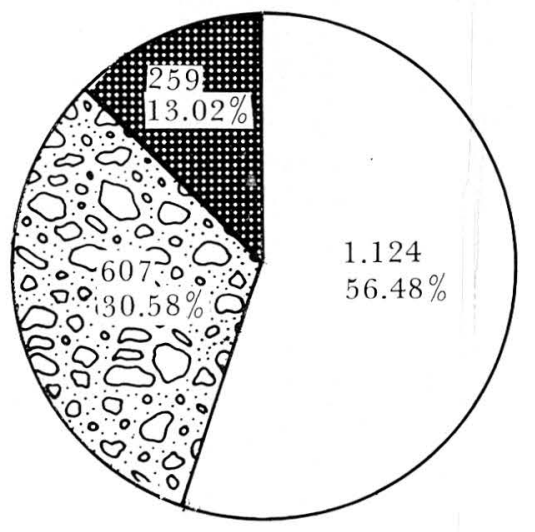

0 - EMBARAZO

$1-4$ EMBARAZOS

$5 y+$ EMBARAZOS

SOBRE 1.990 CASOS INFORMADOS/ 8 SIN DATO

7.8. Distribución de las muertes perinatales según número de mortinatos previos

Las cuatro quintas partes $(80.77 \%)$ no tenían antecedentes de mortinato. El $16.78 \%$ tenían el antecedente de una muerte anteparto (Gráfica 9).

GRAFICA 9

MORTINATOS PREVIOS

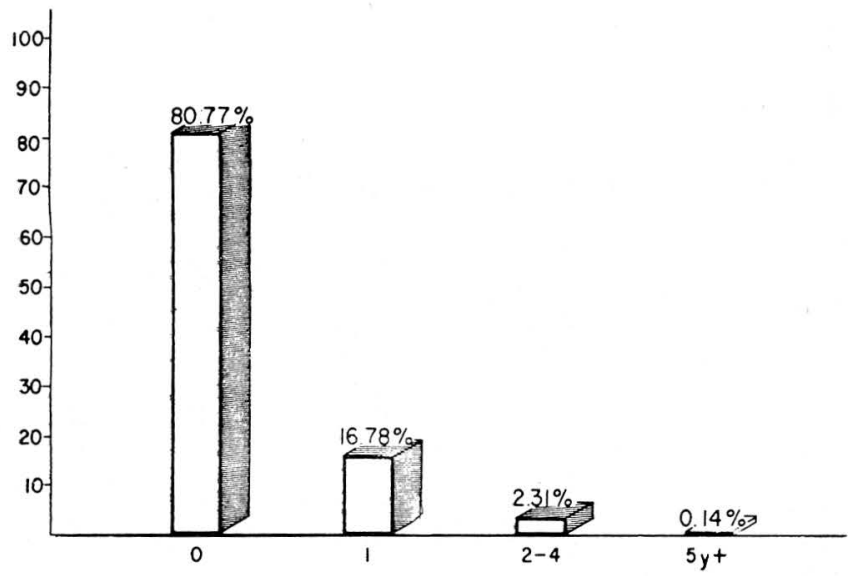

MORTINATOS PREVIOS SOBRE 1.383 CASOS/615 SIN DATO

\subsection{Distribución de las muertes perinatales} según atención prenatal

El 58.81\% tuvieron control prenatal (Mínimo dos consultas de acuerdo con la norma de la división materno infantil de Minsalud) (Gráfica 10).
GRAFICA 10

ATENCION PRENATAL

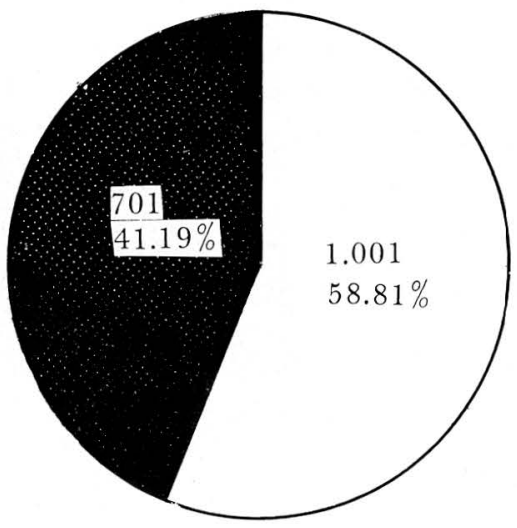

SI

NO

SOBRE 1.702 CASOS INFORMADOS / 296 SIN DATO

7.10. Forma de terminación del embarazo

La gran mayoría fueron partos espontáneos, 1.377 casos (70.94\%); muy pocos casos fueron partos intervenidos, 88 casos $(4.53 \%)$. Una cuarta parte, 476 casos, terminaron en cesárea $(24.52 \%)$. (Gráfica 11).

\section{GRAFICA 11}

FORMA DE TERMINACION DEL EMBARAZO

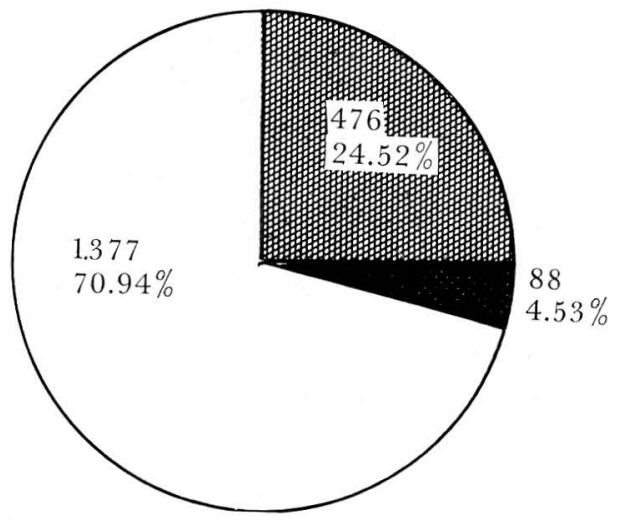

CESAREA

VAGINAL INTERVENIDO

VAGINAL ESPONTANEO

SOBRE 1.941 CASOS INFORMADOS / 57 SIN DATO 


\subsection{Distribución de las muertes perinatales según duración del trabajo de parto}

El mayor número de casos (38.93\%), tuvo trabajo de parto entre 7 y 12 horas; el $12.78 \%$ no tuvo trabajo de parto y en el $3.72 \%$ fue menor de dos horas, (Gráfica 12).

GRAFICA 12

DURACION DEL TRABAJO DE PARTO

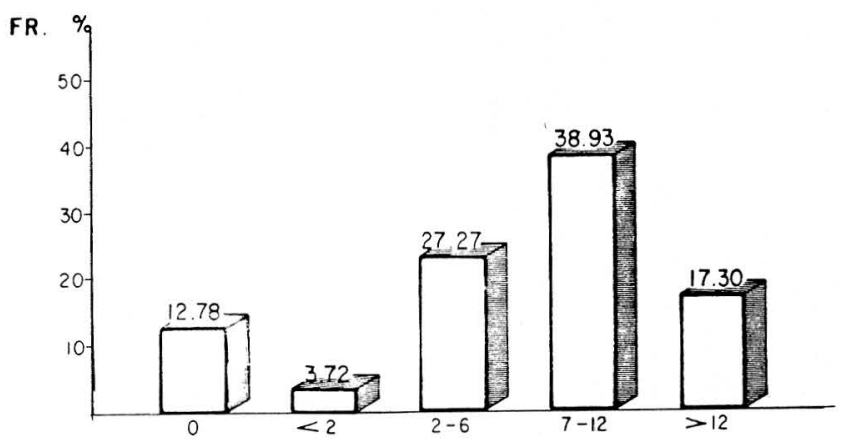

HORAS DE TRABAJO

SOBRE 1.690 CASOS INFORMADOS/308 SIN DATO

\subsection{Muertes perinatales según edad gestacional}

No se encontró mayor diferencia entre los muertos de pretérmino, 915 casos, $50.61 \%$ y los de términos, 829 casos, $45.85 \%$. Solamente $36(1.99 \%)$ se encontraron en post-término, y 28 casos (1.49\%). En menores de 28 semanas incluidos en el análisis por pesos mayores de 1.000 gramos, (Gráfica 13).

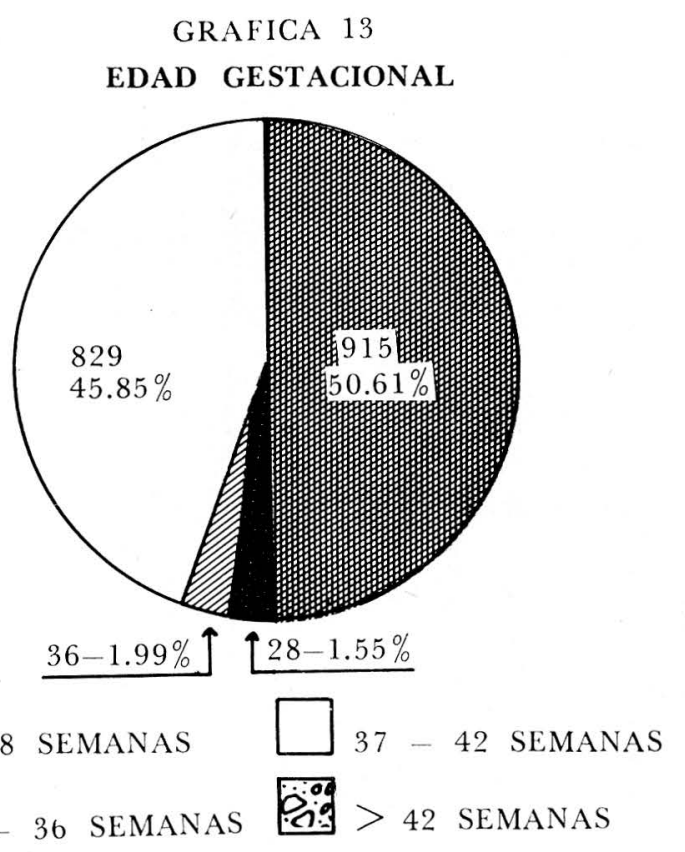

SOBRE 1.807 CASOS INFORMADOS / 191 SIN DATO

\subsection{Mortalidad perinatal según peso al nacer}

Los recién nacidos con muy bajo peso al nacer menor de 1.500 gramos fueron 410 (21.97\%), mientras que los nacidos con un peso de 3.500 gramos o más sólo son 172 casos (9.22\%). Más de la mitad de los casos de mortalidad perinatal $59.86 \%$, nació con peso inferior a 2.500 gramos. La cifra de productos muertos con peso adecuado entre 2.500 y 3.499 gramos, es muy alto, (Gráfica 14).
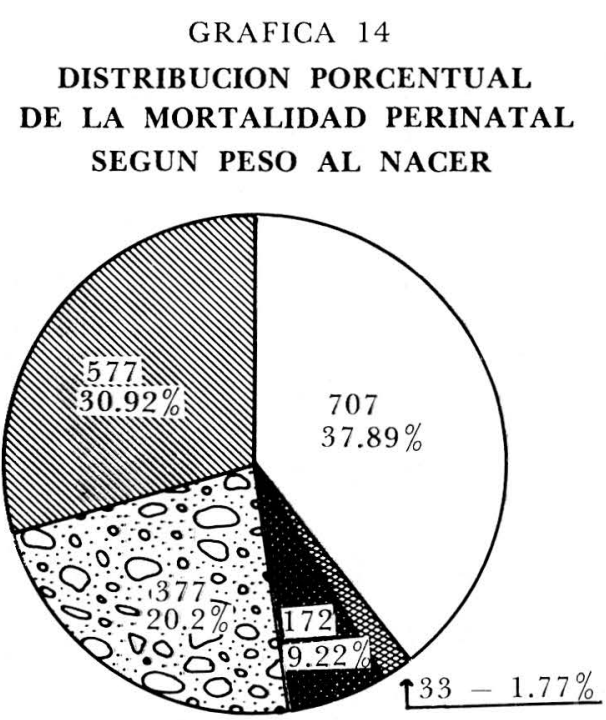

闻被 $<1.000 \mathrm{Grs}$

1.000 Grs. $-1.499^{\circ} \mathrm{Grs}$.

$1.500-2.499$ Grs.

$2.500-3.499 \mathrm{Grs}$.

$>3.500 \mathrm{Grs}$.

SOBRE 1.866 CASOS INFORMADOS - 133 SIN DATO

\subsection{Promedio de peso al nacer según componentes de la mortalidad perinatal}

En todos los componentes el promedio de peso se encontró entre 1.994.20 gramos y más, encontrándose el promedio más alto en el componente intraparto con 2.72.5.50 gramos, (Gráfica 15).

\subsection{Mortalidad perinatal según apgar al minuto y a los cinco minutos}

Dos casos de cada tres tuvieron Apgar de 0 al minuto, es decir, nacieron muertos, y sólo el $8.7 \%$ de los mismos nacieron con un índice de Apgar de 7 y más. Del total de nacidos vivos uno de cada 10 
GRAFICA 15

PROMEDIO DE PESO AL NACER SEGUN COMPONENTES DE LA MORTALIDAD PERINATAL

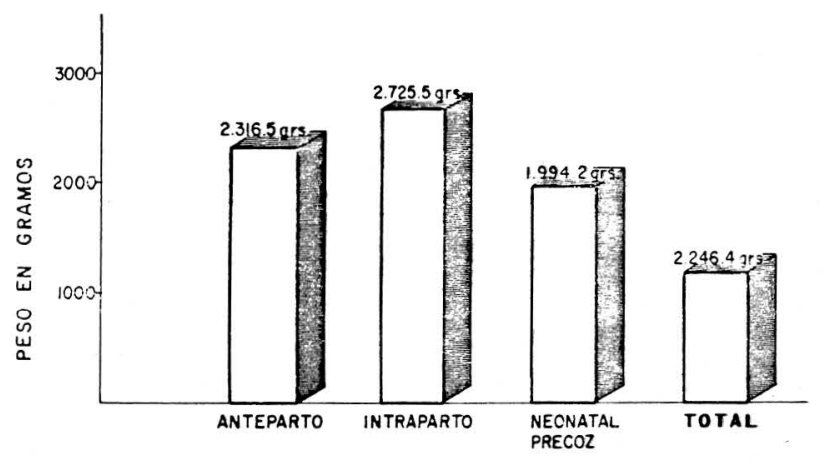

murió antes de cinco minutos; mientras que el $42.5 \%$ tuvieron Apgar en ese momento igual o superior a 7 , lo que nos hace colegir que la causa de muerte estuvo principalmente representada por el S.D.R. (Gráfica 16).

\section{GRAFICA 16}

MORTALIDAD PERINATAL SEGUN APGAR
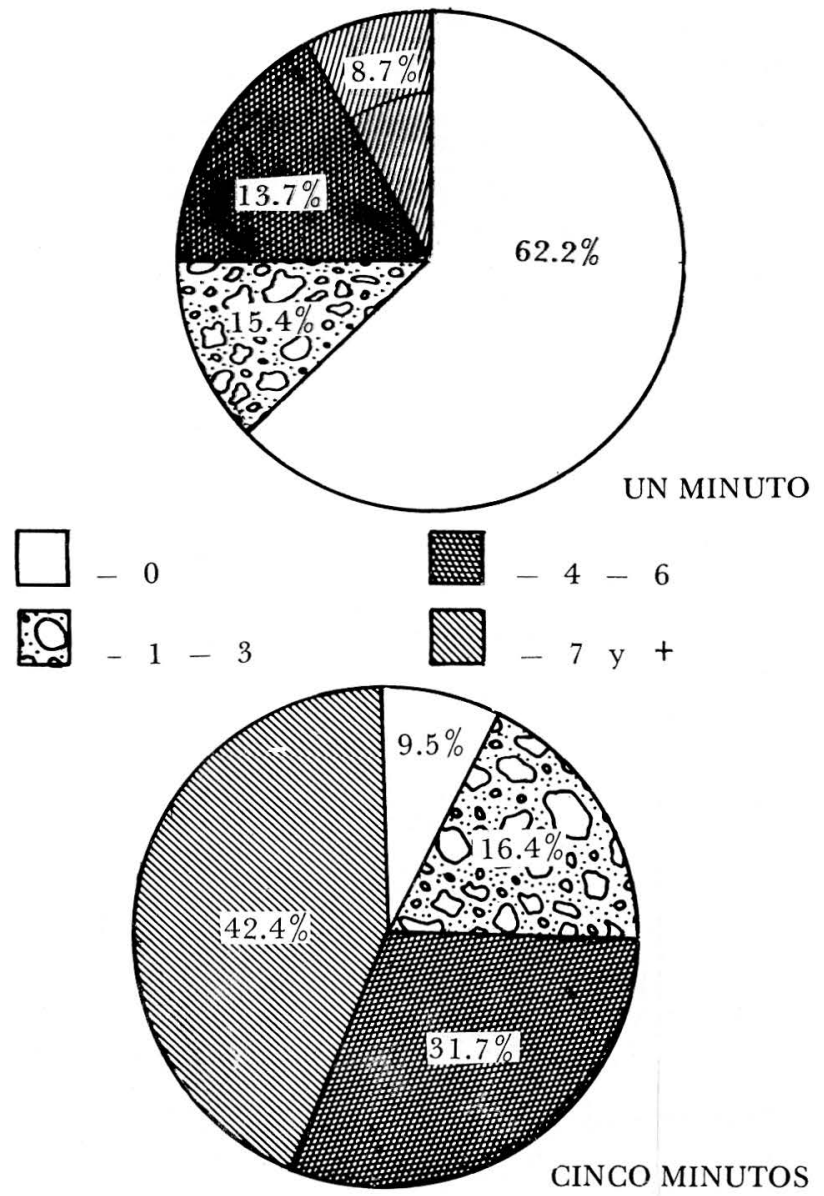

SOBRE 1.770 CASOS INFORMADOS/228 SIN DATO

\subsection{Distribución de las muertes perinatales según sexo}

Se encontró un mayor número de muertos de sexo masculino (56.59\%) lo cual está de acuerdo con la literatura realizada sobre el tema. (Gráfica 17). Al analizar el índice de masculinidad, vemos que por cada 100 mujeres que fallecen mueren 130 hombres (Ver fórmula en Gráfica 17).

\section{GRAFICA 17}

SEXO DEL R.N.

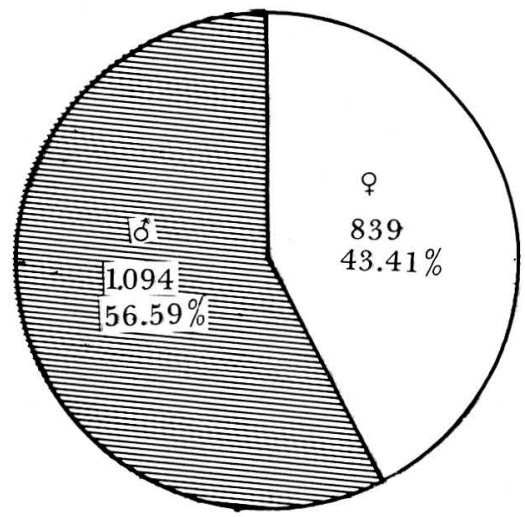

$\mathrm{IM}=\frac{\text { No. } \delta^{\dagger}}{\text { No. } q} \times 100$

$\begin{aligned} & \text { INDICE DE } \\ & \text { MASCULINIDAD }\end{aligned}=\frac{1.094 \delta}{839 \%} \times 100=130.39$

SOBRE 1.933 CASOS INFORMADOS/65 SIN DATO

\subsection{Distribución de la mortalidad perinatal según sus componentes}

Se encuentra el mayor porcentaje $51.45 \%$ en el componente anteparto, siendo menor, pero importante, el porcentaje de muerte in traparto, $12.36 \%$. E1 porcentaje de muerte neonatal precoz fue de $36.19 \%$. (Gráfica 18).

\subsection{Complicaciones del embarazo asociadas con la mortalidad perinatal}

La primera causa está representada por la amenaza de parto pretérmino con $17.82 \%$, seguida de la hipertensión inducida por el embarazo con $16.22 \%$. Le siguen en frecuencia, la ruptura prematura de membranas, $14.31 \%$, el desprendimiento prematuro de placenta $10.36 \%$ y el retardo del crecimiento intrauterino con $10.11 \%$; la diabetes mellitus apenas 
GRAFICA 18

DISTRIBUCION PORGENTUAL DE LA MORTALIDAD PERINATAL SEGUN SUS COMPONENTES

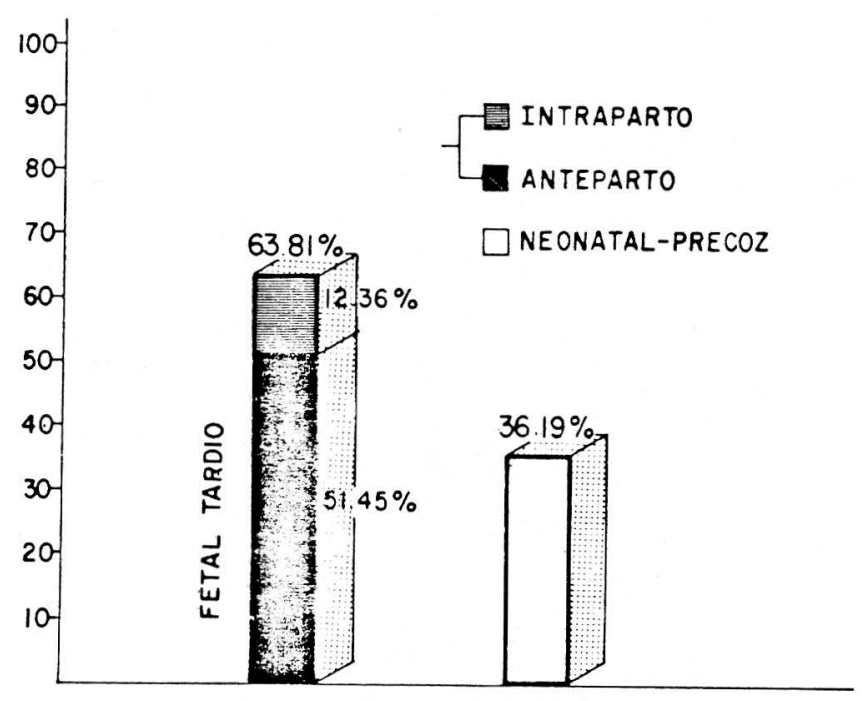

COMPONENTES DE LA MUERTE

ocupó el décimo lugar con $3.55 \%$. Si sumamos la hipertensión crónica a la hipertensión inducida por el embarazo, los fenómenos hipertensivos de la gestación ocupan el primer lugar con $20.72 \%$. (Tabla 4).

Dos de las tres primeras complicaciones asociadas al embarazo corresponden a la amenaza de parto pretérmino (primer lugar) y a la ruptura prematura de membranas (Tercer lugar). (Tabla 4), las cuales a su vez están íntimamente ligadas con la terminación del embarazo antes del término, entidad que ocupa el primer lugar como complicación asociada al parto, (Tabla 5), y con sindrome de dificultad respiratoria que ocupó el segundo lugar dentro de las 999 causas, (Tabla 7) y el tercero èn las complicaciones del recién nacido, (Tabla 6); además el recién nacido pretérmino ocupó el primer lugar en las complicaciones del recién nacido, (Tabla 6$)$.

\subsection{Afecciones maternas concurrentes asociadas con la mortalidad perinatal según sus componentes}

En el componente anteparto una tercera parte de las muertes se debió a la anoxia, 31 casos (31.34\%); el segundo lugar lo ocupó la hipertensión con 237 casos $(23.65 \%)$. En el componente in traparto el primer lugar lo ocupó con más de la cuarta parte de los casos (28.06\%), la R.P.M. y el segundo lugar la hipertensión con $20.92 \%$.

En el componente neonatal precoz el primer lugar, con una tercera parte de los casos con $32.52 \%$ correspondió a la amenaza de parto pretérmino y el segundo lugar a la R.P.M. con $22.55 \%$.

Las tres primeras causas en los tres componentes fueron en su orden la hipertensión con $395 \mathrm{ca}-$ sos $(21.82 \%)$, la amenaza de parto pretérmino con $343(18.95 \%)$ y la muerte fetal por anoxia 342 $(18.90 \%)$. (Gráfica 19).

TABLA 4

COMPLICACIONES DEL EMBARAZO ASOCIADAS CON LA MORTALIDAD PERINATAL

\begin{tabular}{|c|c|c|c|}
\hline ORDEN & COMPLICACION & No. & $\%(*)$ \\
\hline 10. & Amenaza de parto pretérmino & 356 & 17.82 \\
\hline 20. & Hipertensión inducida por el embarazo & 324 & 16.22 \\
\hline 3o. & Ruptura prematura de membranas & 286 & 14.31 \\
\hline 40. & Desprendimiento prematuro de la Placenta & 207 & 10.36 \\
\hline 50. & Retardo del crecimiento fetal Intrauterino & 202 & 10.11 \\
\hline 60. & Gran multiparidad & 131 & 6.56 \\
\hline 70. & Corioamnionitis & 112 & 5.61 \\
\hline 80. & Embarazo múltiple & 99 & 4.95 \\
\hline 90. & Hipertensión crónica & 90 & 4.50 \\
\hline 10 o. & Diabetes Mellitus & 71 & 3.55 \\
\hline
\end{tabular}


GRAFICA 19

AFECCIONES MATERNAS CONCURRENTES ASOCIADAS CON LA MORTALIDAD PERINATAL SEGUN SUS COMPONENTES

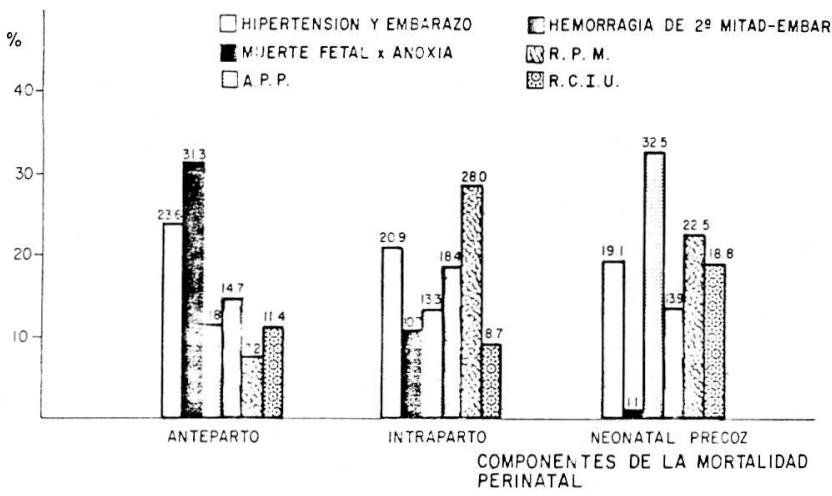

7.20. Complicaciones del parto asociadas con la mortalidad perinatal

Cuatro de cada 10 complicaciones corresponden al parto prematuro $(42.09 \%)$ duplicando al parto por cesárea que ocupó el segundo lugar con $(21.87 \%)$. (Tabla 5).

7.21. Alteraciones del parto, asociadas con la mortalidad perinatal según sus componentes

El parto pretérmino ocupó el primer lugar en los 3 componentes con $48.1 \%$ en el anteparto, $31.8 \%$ en el intraparto y $52.9 \%$ en el neonatal precoz. La cesárea ocupó el segundo lugar en los 3 componentes con $17.1 \%$ en el anteparto, $24.0 \%$ en el intrapar- to y $28.6 \%$ en el neonatal precoz. La compresión del cordón ocupó el tercer lugar en los 3 componentes con $16.4 \%$ en el anteparto, $19.8 \%$ en el intraparto y $9.2 \%$ en el neonatal precoz. (Gráfica 20).

\subsection{Duración del trabajo de parto según componentes de la mortalidad perinatal}

La duración del trabajo de parto podría ser un factor influyente en la mortalidad neonatal precoz y en la intraparto; en la intraparto se observa que un trabajo mayor de doce horas aporta la mayor proporción de casos. En el componente neonatal precoz se aprecia una tendencia decreciente a medida que aumenta tiempo de trabajo de parto y la pre-

GRAFICA 20

\section{ALTERACIONES DEL PARTO ASOCIADAS CON LA MORTALIDAD PERINATAL SEGUN SUS COMPONENTES}

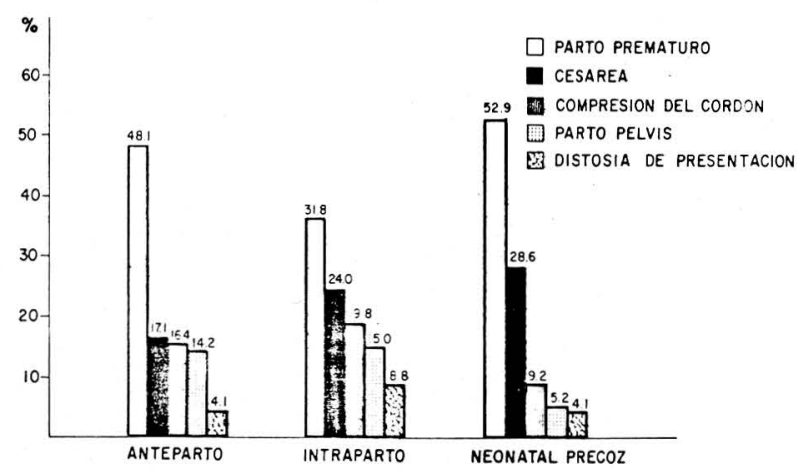

TABLA 5

COMPLICACIONES DEL PARTO ASOCIADAS CON LA MORTALIDAD PERINATAL

\begin{tabular}{|clrr|}
\hline ORDEN & COMPLICACION & No. \\
\hline 10. & Parto Prematuro & 841 & 42.09 \\
20. & Parto por cesárea & 437 & 21.87 \\
30. & Parto de Pelvis & 231 & 11.56 \\
40. & Compresión del Cordón & 202 & 10.11 \\
50. & Parto con Fórceps & 83 & 4.15 \\
60. & Distocia de presentación & 82 & 4.15 \\
70. & Prolapso de cordón & 70 & 4.10 \\
80. & Trabajo de parto prolongado & 4.50 & 2.15 \\
90. & Hiperdinamia e Hipertonia & 42 & 2.10 \\
\hline
\end{tabular}


sencia de la cesárea como principal hecho que acompaña al componente neonatal precoz. (Todos los casos sin trabajo de parto y posiblemente todos los casos con duración de dos horas), se explica en el hecho de que dicha intervención se realiza cuando existe ya un severo compromiso materno o del recién nacido por un S.F.A. (Gráfica 21).

GRAFICA 21

DURACION DEL TRABAJO

DE PARTO SEGUN COMPONENTES

DE LA MORTALIDAD PERINATAL

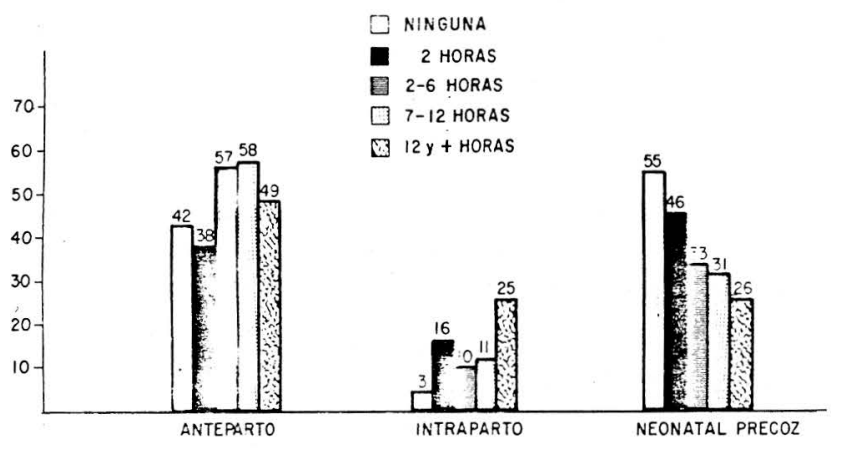

SOBRE 1690 CASOS INFORMADOS - 308 SIN DATO

\subsection{Tipo de presentación según componente de la mortalidad perinatal}

La presentación cefálica es mayoría en el componente anteparto (53\%), en cambio las presentaciones distócicas son mayoría en los componentes intraparto y neonatal precoz. La presentación "Otra", son presentaciones incompatibles con la vía vaginal y por tanto la vía del nacimiento fue la cesárea, la cual es mayoría en los componentes in traparto y neonatal precoz. (Gráfica 22).

\subsection{Tipo de parto según componentes de la mortalidad perinatal}

Dentro de la mortalidad anteparto, llama la atención la proporción de cesáreas, pues como se observa, de 1.001 casos de muerte anteparto, 151 $(15.08 \%)$ fueron intervenidos, posiblemente por complicaciones obstétricas independientes del producto y que obligaron a la intervención con feto muerto. En las 2/3 partes de los casos del componente intraparto y en más de la mitad de los casos del componente neonatal precoz el tipo de parto fue vaginal espontáneo $(63.3 \%$ y $58.4 \%$ respectivamente). (Gráfica 23).

\section{GRAFICA 22}

\section{TIPO DE PRESENTACION SEGUN COMPONENTES DE LA MORTALIDAD PERINATAL}

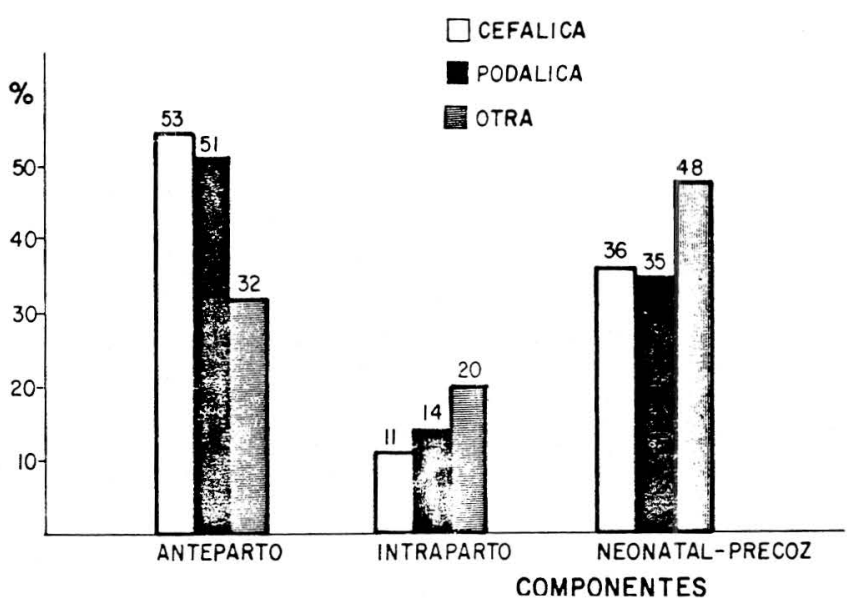

SOBRE 1.491 CASOS INFORMADOS/507 SIN DATO

GRAFICA 23

TIPO DE PARTO SEGUN COMPONENTES DE LA MORTALIDAD PERINATAL

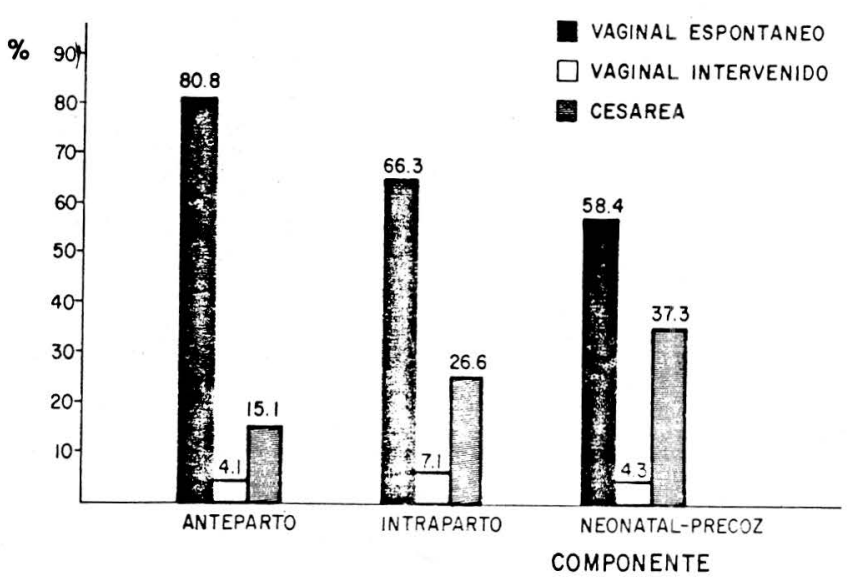

SOBRE 1.941 CASOS INFORMADOS/57 SIN DATO

\subsection{Complicaciones del recién nacido asociadas con la mortalidad perinatal}

La tercera parte de las complicaciones del recién nacido asociadas con la muerte perinatal la ocupa el recién nacido pretérmino (32.73\%). Seguido por el sufrimiento fetal con $17.11 \%$ y el sindrome de dificultad respiratoria con $13.16 \%$. Es de anotar bajo un porcentaje para hemorragia cerebral y trauma obstétrico. (Tabla 6). 
TABLA 6

COMPLICACIONES DEL RECIEN NACIDO ASOCIADAS

CON LA MORTALIDAD PERINATAL

\begin{tabular}{|c|c|c|c|}
\hline ORDEN & COMPLICACION & No. & $\%(*)$ \\
\hline 10. & Pretérmino & 654 & 32.73 \\
\hline 20. & Sufrimiento fetal & 342 & 17.11 \\
\hline 30. & Sindrome defic. respiratoria & 265 & 13.26 \\
\hline 40. & Malformaciones congénitas & 231 & 11.56 \\
\hline 50. & Sepsis in-útero o neonatal & 176 & 8.80 \\
\hline 60. & Aspiración de meconio & 111 & 5.56 \\
\hline 70. & Hemorragia cerebral & 69 & 3.45 \\
\hline 80. & Trauma obstétrico & 62 & 3.10 \\
\hline 90. & Post - término & 44 & 2.20 \\
\hline 100. & Hemorragia pulmonar & 42 & 2.10 \\
\hline
\end{tabular}

(*) SOBRE EL TOTAL DE MUERTES: 1.998

\subsection{Afecciones del neonato asociadas con la} mortalidad perinatal según componentes

El parto pretérmino es mayoría en los 3 componentes con $91.3 \%$ del anteparto, $61.10 \%$ del intraparto y $38 \%$ del neonatal precoz.

La segunda causa del componente intraparto es el sufrimiento fetal durante el trabajo con $18.52 \%$ y en la neonatal precoz el S.D.R. del recién nacido con $24.64 \%$. (Gráfica 24).

GRAFICA 24

AFECCIONES DEL NEONATO

ASOCIADAS CON LA MORTALIDAD

PERINATAL SEGUN SUS COMPONENTES

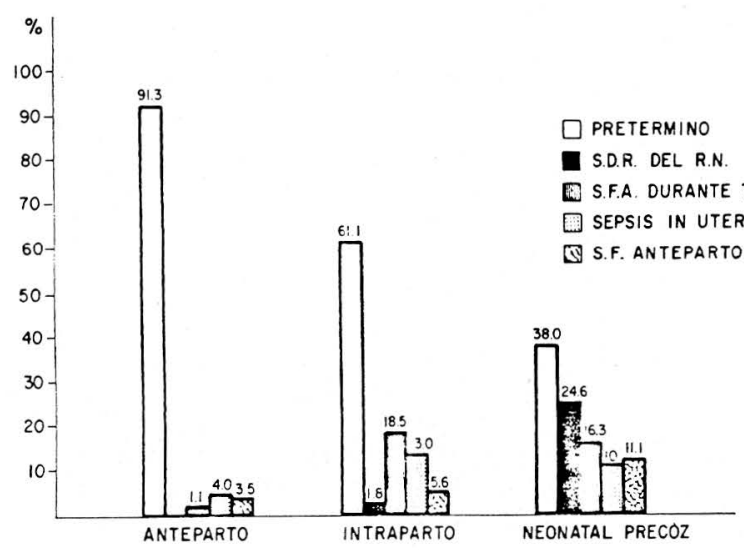

\subsection{Autopsias realizadas}

Al $81.24 \%$ de las muertes no se les practicó autopsia, (Gráfica 25); esto impide conocer con seguridad, la verdadera etiología de la muerte.

GRAFICA 25

\section{AUTOPSIAS REALIZADAS}

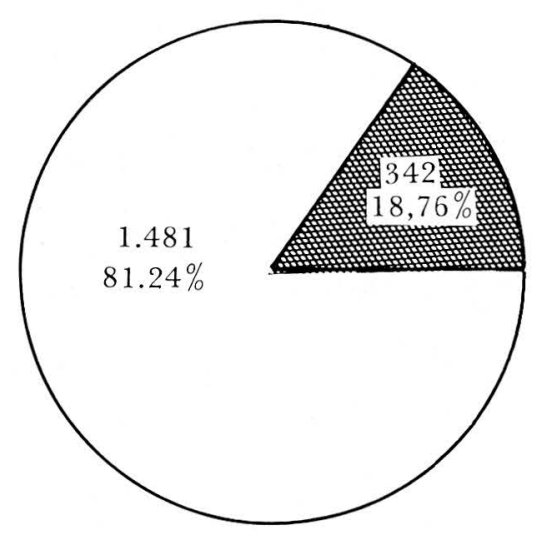

SI

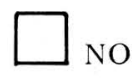

SOBRE 1.823 CASOS INFORMADOS / 175 SIN DATO

7.28. Primeras causas de mortalidad perinatal (según clasificación internacional de 999 causas)

La primera causa de muerte perinatal (anoxia anteparto) representa más de la cuarta parte del total. Las cinco primeras representan la mayoría con 
$61.87 \%$, ocupando el sindrome de dificultad respiratoria y el desprendimiento prematuro de la placenta en segundo y tercer lugar respectivamente. (Tabla 7). En el diagnóstico etiológico de la anoxia anteparto no es posible identificarlo con seguridad y puede ser el resultado más bien, del desconocimiento de la causa de muerte. El S.D.R. sigue siendo una etiología muy importante en la mortalidad perinatal (segundo lugar); y el tercer lugar del abruptio de placenta, se relaciona bien con la hipertensión inducida por el embarazo que ocupa el segundo lugar en la tabla de las complicaciones asociadas a la gestación. (Tabla 7).

\subsection{Primeras causas de mortalidad perinatal según componentes}

La anoxia anteparto y el desprendimiento prematuro de la placenta que dará como resultado la anoxia, ocupan el primero y segundo lugar del componente anteparto. El prolapso de cordón ocupa el primer lugar en el período intraparto, seguido del desprendimiento prematuro de la r'acenta y en el tercer lugar la compresión del cordon. Si sumamos las alteraciones del cordón hacen el $60.9 \%$ de los casos del componente intraparto. La cifra elevada de “otras causas" en el componente intraparto (57.4\%) indica gran dispersión de las mismas en este período. En el neonatal precoz surgen las causas propias de la prematuridad y del inadecuado manejo perinatal, como son el S.D.R., la sepsis neonatal, y la hemorragia cerebral entre las más importantes, (Tabla 8$)$.

\subsection{Primeras causas de muerte perinatal según edad de la madre}

En los tres grupos de edad la causa más frecuente es la anoxia anteparto, $20.23 \%$ en 18 años u menos; $26.95 \%$ en 19 a 34 años y $29.17 \%$ en 35 años y más. Descartando la anoxia anteparto cuyo valor etiológico no es muy seguro, el abruptio ocupa el primer lugar en el grupo de 18 años y menos con $18.50 \%$ y en el grupo de 19 a 34 años con $14.61 \%$. El segundo lugar en el grupo de 35 años y más, $7.81 \%$, donde la causa más frecuente fue el sindrome de dificultad respiratoria del recién nacido con $12.50 \%$. (Gráfica 26).

\subsection{Primeras causas de mortalidad perinatal según atención prenatal}

En la Tabla 9 se encuentran las cinco primeras causas de muerte perinatal en orden de frecuencia, en las pacientes con y sin control prenatal. Las alteraciones del cordón ocupan el primer lugar en los dos grupos y no guardan relación con un buen o mal control prenatal.

TABLA 7

\section{PRIMERAS CAUSAS DE MORTALIDAD PERINATAL SEgun 999 CAUSAS}

\begin{tabular}{|clcr|}
\hline \multicolumn{1}{c|}{ CAUSAS } & No. & $\%$ \\
\hline & Muerte fetal por anoxia ante-parto & 399 & 28.41 \\
1. & Sindrome de dificultad respiratoria & 214 & 15.24 \\
& del recién nacido & 108 & 7.69 \\
3. & Desprendimiento prematuro de la placenta & 80 & 5.69 \\
4. & Sepsis in útero o neonatal & 68 & 4.84 \\
5. & Compresión de cordón, nudo o circular & 57 & 4.05 \\
6. & Aspiración de meconio & 53 & 3.77 \\
7. & Hemorragia cerebral & 39 & 2.77 \\
8. & Sufrimiento fetal diagnosticado durante & 34 & 2.42 \\
& el trabajo de parto en recién nacido vivo & 30 & 2.13 \\
\hline Prolapso de cordón & Corioamnionitis & 1.082 & 77.06 \\
\hline & SUB - TOTAL & 322 & 22.94 \\
\hline
\end{tabular}


TABLA 8

PRIMERAS CAUSAS DE MORTALIDAD PERINATAL SEGUN COMPONENTES

\begin{tabular}{|c|c|c|c|c|c|c|}
\hline ORDEN & ANTEPARTO & $\%$ & INTRAPARTO & $\%$ & $\begin{array}{l}\text { NEONATAL } \\
\text { PRECOZ }\end{array}$ & $\%$ \\
\hline 10. & Anoxia anteparto & 52.8 & Prolapso cordón & 12.9 & S.D.R. & 34.0 \\
\hline 20. & Abruptio & 11.5 & Abruptio & 11.3 & Sepsis neonatal & 11.7 \\
\hline 30. & Compresión del & & & & & \\
\hline & cordón & 6.8 & Compresión cordón & 8.1 & Hemorragia cerebral & 7.7 \\
\hline 40. & Corioamnionitis & 2.2 & Corioamnionitis & 6.5 & Aspiración meconio & 6.7 \\
\hline 50. & Aspiración meconio & 1.4 & S.F.A. & 3.8 & S.F.A. & 4.9 \\
\hline & OTRAS & 25.3 & OTRAS & 57.4 & OTRAS & 35.0 \\
\hline
\end{tabular}

SOBRE 1.510 CASOS INFORMADOS / SIN DATO 488

GRAFICA 26

CAUSAS DE MORTALIDAD PERINATAL SEGUN EDAD MATERNA

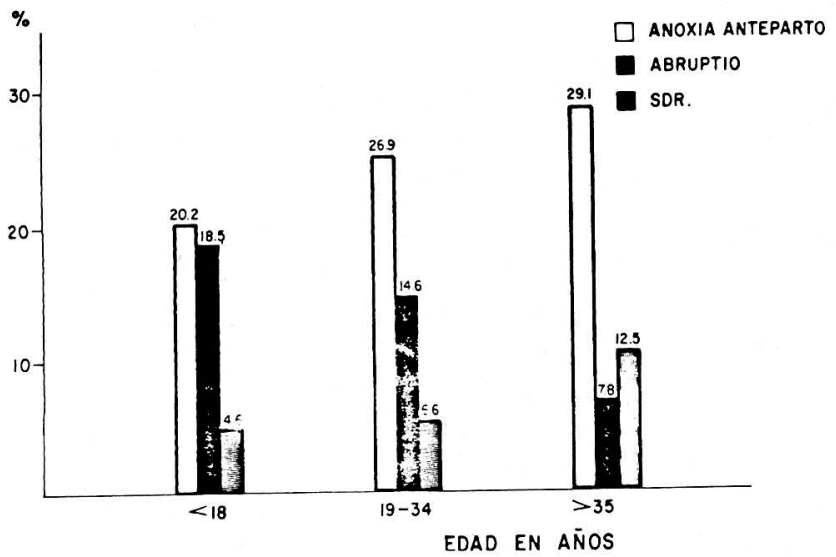

7.32. Primeras causas de mortalidad perinatal según edad gestacional

Dentro del período pretérmino, el S.D.R. y la anoxia anteparto constituyen casi la mitad de los casos. En el período de término sobresale especialmente la anoxia anteparto, toda vez que uno de cada tres casos se debieron a ella. En post-término la mayor cantidad de casos fueron debidos a la aspiración de meconio. (Tabla 10).

\subsection{Primeras causas de mortalidad perinatal según tipo de presentación}

La causa que prevalece en primer lugar es la anoxia anteparto, siendo la primera en los tres tipos de presentación y aportando una quinta parte de las muertes. Sigue en orden de prevalencia el

TABLA 9

PRIMERAS GAUSAS DE MORTALIDAD PERINATAL SEGUN ATENCION PRENATAL

\begin{tabular}{|c|c|c|}
\hline ORDEN & CON ATENCION PRENATAL & SIN ATENCION PRENATAL \\
\hline 10. & Compresión de cordón & Prolapso de cordón \\
\hline 20. & Hemorragia cerebral & $\begin{array}{l}\text { Desprendimiento prematuro de la } \\
\text { placenta }\end{array}$ \\
\hline 3o. & Aspiración de meconio & Sepsis in útero o neonatal \\
\hline 40. & $\begin{array}{l}\text { Sindrome de deficiencia respiratoria } \\
\text { del recién nacido }\end{array}$ & $\begin{array}{l}\text { Sufrimiento fetal intraparto en el } \\
\text { recién nacido vivo }\end{array}$ \\
\hline 50. & Anoxia anteparto & Aspiración meconio \\
\hline
\end{tabular}


TABLA 10

PRIMERAS CAUSAS DE MORTALIDAD PERINATAL SEGUN EDAD GESTACIONAL

\begin{tabular}{|c|c|c|c|c|c|c|}
\hline ORDEN & $\begin{array}{l}\text { PRETERMINO } \\
\text { (28-36 SEM) }\end{array}$ & $\%$ & $\begin{array}{l}\text { TERMINO } \\
(37-42 \text { SEM })\end{array}$ & $\%$ & $\begin{array}{l}\text { POST-TERMINO } \\
\text { (MAS DE } 42 \text { SEM) }\end{array}$ & $\%$ \\
\hline 10. & S.D.R. & 23.9 & Anoxia anteparto & 32.0 & Aspiración meconio & 22.2 \\
\hline 20. & Anoxia anteparto & 22.9 & Abruptio & 7.9 & S.F.A. & 7.4 \\
\hline 30. & Sepsis in vitro & 6.7 & Aspiración meconio & 6.3 & Sepsis in vitro & 3.7 \\
\hline 40. & Abruptio & 6.1 & Compresión de cordón & 6.1 & Compresión cordón & 3.7 \\
\hline 50. & Hemorragia cerebral & 4.6 & S.F.A. & 4.5 & Prolapso cordón & 3.7 \\
\hline & Otros & 35 & Otros & 43.2 & Otros & 59.3 \\
\hline
\end{tabular}

SOBRE 1.353 CASOS INFORMADOS / SIN DATO 157

S.D.R. que ocupa el segundo lugar en los tres tipos de presentación y que aporta una proporción similar de muertes en cada uno de los tres tipos de presentación. (Tabla 11).

\subsection{Primeras causas de mortalidad perinatal según tipo de parto}

La anoxia anteparto ocupa el primer lugar como causa de muerte cuando el parto es vaginal, ya sea espontáneo (31.4\%), o intervenido (31.80\%). Cuando el producto es extraído vía abdominal, el S.D.R. ocupa el primer lugar $(16.80 \%)$ desplazando a la anoxia anteparto. (Tabla 12).

\subsection{Primeras causas de mortalidad perinatal según duración del trabajo de parto}

Se observa que cuando no existe trabajo de parto o éste es menor de dos horas, la quinta parte de los casos los aporta el S.D.R.; en la medida en que transcurre el tiempo de trabajo de parto, la ano-xia anteparto ocupa el primer puesto, pasando el S.D.R. a un segundo lugar, hasta cuando el tiempo es menor de 12 horas. Cuando éste es, igual o mayor a doce horas el S.D.R. es desplazado por causas propias de un proceso prolongado, como son la compresión de cordón umbilical y la aspiración de meconio, secundaria al sufrimiento fetal. (Tạbla 13).

TABLA 11

\section{PRIMERAS CAUSAS DE MORTALIDAD PERINATAL SEGUN TIPO DE PRESENTACION}

\begin{tabular}{|c|c|c|c|c|c|c|}
\hline ORDEN & CEFALICA & $\%$ & PELVIANA & $\%$ & OTRA & $\%$ \\
\hline 10. & Anoxia anteparto & 27.0 & Anoxia anteparțo & 26.67 & Anoxia anteparto & 21.28 \\
\hline 20. & $\begin{array}{l}\text { Sindrome deficiencia } \\
\text { respiratoria }\end{array}$ & 13.79 & $\begin{array}{l}\text { Sindrome deficiencia } \\
\text { respiratoria }\end{array}$ & 14.51 & $\begin{array}{l}\text { Sindrome deficiencia } \\
\text { respiratoria }\end{array}$ & 19.15 \\
\hline 30. & Abruptio & 8.33 & $\begin{array}{l}\text { Compresión de } \\
\text { cordón }\end{array}$ & 4.71 & Hemorragia cerebral & 8.51 \\
\hline 40. & $\begin{array}{l}\text { Sepsis in útero o } \\
\text { neonatal }\end{array}$ & 5.72 & Prolapso de cordón & 4.71 & Prolapso cordón & 3.51 \\
\hline \multirow[t]{3}{*}{50.} & $\begin{array}{l}\text { Compresión de } \\
\text { cordón }\end{array}$ & 4.54 & Hemorragia cerebral & 4.71 & Sepsis in útero & 6.38 \\
\hline & & & & & Aspiración meconio & 6.38 \\
\hline & Otras & 40.62 & Otras & 44.69 & Otras & 29.79 \\
\hline
\end{tabular}


TABLA 12

PRIMERAS CAUSAS DE MORTALIDAD PERINATAL

SEGUN TIPO DE PARTO

\begin{tabular}{|c|c|c|c|c|c|c|}
\hline ORDEN & $\begin{array}{c}\text { VAGINAL } \\
\text { ESPONTANEO }\end{array}$ & $\%$ & $\begin{array}{c}\text { VAGINAL } \\
\text { INTERVENIDA }\end{array}$ & $\%$ & CESAREA & $\%$ \\
\hline 10. & Anoxia anteparto & 31.4 & Anoxia anteparto & 31.8 & S.D.R. & 16.8 \\
\hline 20. & S.D.R. & 13.4 & Compresión cordón & 9.5 & Anoxia anteparto & 12.8 \\
\hline 30. & Abruptio & 5.8 & S.D.R. & 7.9 & Abruptio & 12.5 \\
\hline 40. & Compresión cordón & 5.2 & Hemorragia cerebral & 7.9 & Sepsis in útero & 7.5 \\
\hline \multirow[t]{2}{*}{50.} & Sepsis in útero & 4.6 & Prolapso cordón & 3.2 & Aspiración del meconio & 6.1 \\
\hline & & & Sepsis in útero & 3.2 & & \\
\hline & Otras & 39.6 & Otras & 36.5 & Otras & 44.3 \\
\hline
\end{tabular}

SOBRE 1.479 CASOS INFORMADOS / SIN DATO 519

TABLA 13

PRIMERAS CAUSAS DE MORTALIDAD PERINATAL SEGUN DURACION DE TRABAJO DE PARTO

\begin{tabular}{|c|c|c|c|c|c|c|c|c|c|c|}
\hline ORDEN & N NINGUNA & $\%$ & $\begin{array}{l}\text { MENOS DE } \\
2 \text { HORAS }\end{array}$ & $\%$ & 2-6 HORAS & $\%$ & $7-12$ HORAS & $\%$ & $\begin{array}{l}12 \text { y MAS } \\
\text { HORAS }\end{array}$ & $\%$ \\
\hline 10. & S.D.R. & 21.3 & S.D.R. & 22.0 & $\begin{array}{l}\text { Anoxia ante- } \\
\text { parto }\end{array}$ & 30.2 & $\begin{array}{l}\text { Anoxia ante- } \\
\text { parto }\end{array}$ & 31.5 & $\begin{array}{l}\text { Anoxia } \\
\text { anteparto }\end{array}$ & 25.7 \\
\hline 20. & Abruptio & 15.5 & $\begin{array}{l}\text { Anoxia ante- } \\
\text { parto }\end{array}$ & 14.0 & S.D.R. & 15.6 & S.D.R. & 10.9 & $\begin{array}{l}\text { Compresión } \\
\text { cordón }\end{array}$ & 8.6 \\
\hline 30. & $\begin{array}{l}\text { Anoxia ante- } \\
\text { parto }\end{array}$ & 13.9 & Abruptio & 10.0 & Abruptio & 7.8 & Sep. in útero & 6.6 & $\begin{array}{l}\text { Aspiración } \\
\text { meconio }\end{array}$ & 7.1 \\
\hline 40. & $\begin{array}{l}\text { Sepsis in úte- } \\
\text { ro. }\end{array}$ & 8.1 & $\begin{array}{l}\text { Aspiración } \\
\text { meconio }\end{array}$ & 6.0 & $\begin{array}{l}\text { Sepsis in } \\
\text { útero. }\end{array}$ & 3.8 & $\begin{array}{l}\text { Compresión } \\
\text { cordón }\end{array}$ & 6.2 & S.D.R. & 6.1 \\
\hline & Otras & 38.2 & Otras & 48.0 & Otras & 42.6 & Otras & 44.8 & Otras & 46.4 \\
\hline
\end{tabular}

SOBRE 1.282 CASOS INFORMADOS / SIN DATO 716

\subsection{Primeras causas de mortalidad perinatal según peso al nacer}

Definitivamente las causas que tienen relación con el S.D.R. y la anoxia anteparto son los principales participantes en los casos de mortalidad, alrededor del $45 \%$ para los grupos de $1.000-1.499$ y 1.500 a 2.499. La sepsis, la hemorragia cerebral y el abruptio le siguen en orden de importancia para estos rangos de peso.

En los casos de mortalidad, con peso de 2.500 gramos - 3.499 gramos, el S.D.R. desaparece como causa prioritaria y surgen la anoxia anteparto, el abruptio, y la sepsis in útero o neonatal, ocupando el $50 \%$ de los casos.
En el grupo de 3.500 gramos o más, aparecen como causas prioritarias la anoxia anteparto y la aspiración de meconio ya que en este grupo de S.F.A. ocasionado por el trabajo de parto prolongado es frecuente, dando como resultado las causas ya mencionadas. (Tabla 14).

\subsection{Causa de mortalidad perinatal según la alteración del parto}

La anoxia anteparto se asoció en más de la mitad de los casos $58.71 \%$, al parto prematuro, con las demás alteraciones no existe casi diferencia. El abruptio se asoció en más de la mitad de los casos, $65.76 \%$ con el parto prematuro y casi en la cuarta parte, $20.62 \%$ con la cesárea. Sumadas estas dos al- 
TABLA 14

PRIMERAS CAUSAS DE MORTALIDAD PERINATAL SEGUN PESO AL NACER

\begin{tabular}{|c|c|c|c|c|c|c|c|c|}
\hline ORDEN & MENOR DE 1500 & $\%$ & $1500-2499$ & $\%$ & $2500-3499$ & $\%$ & 3500 y MAS & $\%$ \\
\hline 10. & S.D.R. & 30.2 & $\begin{array}{l}\text { Anoxia ante- } \\
\text { parto. }\end{array}$ & 28.1 & $\begin{array}{l}\text { Anoxia ante- } \\
\text { parto. }\end{array}$ & 33.0 & $\begin{array}{l}\text { Anoxia ante- } \\
\text { parto. }\end{array}$ & 26.9 \\
\hline 20. & Anoxia anteparto & 16.0 & S.D.R. & 16.0 & Abruptio & 9.1 & $\begin{array}{l}\text { Aspiración } \\
\text { meconio }\end{array}$ & 11.9 \\
\hline 30. & Sepsis in útero & 7.7 & Abruptio & 7.0 & Sepsis & 7.4 & Abruptio & 8.2 \\
\hline 40. & Abruptio & 4.7 & $\begin{array}{l}\text { Sepsis in } \\
\text { útero. }\end{array}$ & 6.9 & $\begin{array}{l}\text { Compresión } \\
\text { cordón }\end{array}$ & 6.5 & $\begin{array}{l}\text { Compresión } \\
\text { cordón }\end{array}$ & 6.0 \\
\hline 50. & Hemorragia cerebral & 4.1 & $\begin{array}{l}\text { Hemorragia } \\
\text { cerebral }\end{array}$ & 4.4 & $\begin{array}{l}\text { Hemorragia } \\
\text { cerebral }\end{array}$ & 4.5 & $\begin{array}{l}\text { Corioamnio- } \\
\text { nitis. }\end{array}$ & 3.7 \\
\hline & Otras & 37.3 & Otras & 37.6 & Otras & 39.5 & Otras & 43.3 \\
\hline
\end{tabular}

SOBRE 1.459 CASOS INFORMADOS / SIN DATO 539

teraciones, contituyen el $86.38 \%$ asociadas al abruptio. La sepsis in útero o neonatal en más de la mitad de los casos, $59.34 \%$, se asoció al parto prematuro. (Gráfica 27).

\section{GRAFICA 27}

PRIMERAS CAUSAS DE MORTALIDAD PERINATAL SEGUN LA ALTERACION DEL PARTO

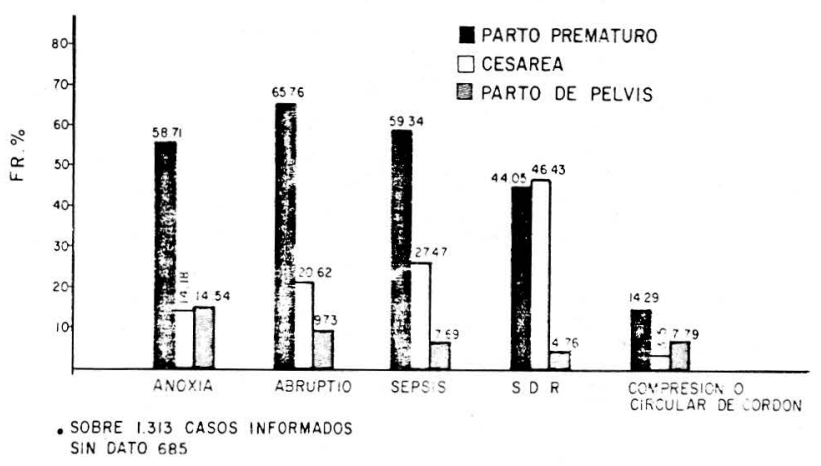

- SOBRE 1.313 CASOS INFORMADOS SIN DATO 685

\subsection{Primeras causas de mortalidad perinatal según las afecciones del neonato}

Las tres primeras causas de muerte perinatal fueron el abruptio con 408, la sepsis con 151 y la hemorragia cerebral con 64 casos.

El abruptio se asoció en casi la mitad de los casos con el recién nacido pretérmino (40.9\%) y con S.D.R. (41.10\%).
Un poco más de la cuarta parte de la sepsis se asoció al R.N. pretérmino (29.1\%). Casi la mitad de las hemorragias cerebrales, $(40.6 \%)$, se asociaron al $\mathrm{R}: \mathrm{N}$. pretérmino y una cuarta parte de las mismas se asociaron con el S.F.A. en el trabajo de parto, $(25 \%)$.

Más de las tres cuartas partes del S.F.A. con R.N. vivo se asoció con la misma patología durante el trabajo de parto (76.7\%). Dos terceras partes de la anoxia anteparto $(76.1 \%)$ se asoció al R.N. pretérmino.

Un poco más de la mitad de los casos que presentaron aspiración de meconio estuvo precedida por el S.F.A. durante el trabajo de parto (59.52\%). (Gráfica 28).

\subsection{Distribución de las autopsias realizadas según componente de la mortalidad perinatal}

De los 342 casos en los cuales se realizó la autopsia, la proporción fue casi igual en el anteparto con 151 casos $(44.15 \%)$ y en el neonatal precoz, con 153 casos (44.74\%). El componente en el cual se realizaron menos autopsias, $38(11.11 \%)$, fue en el intraparto, pero teniendo en cuenta que este componente fue el que menos muertos aportó. (Gráfica 29). 
GRAFICA 28

PRIMERAS CAUSAS DE MORTALIDAD PERINATAL SEGUN LAS AFEGGIONES DEL NEONATO

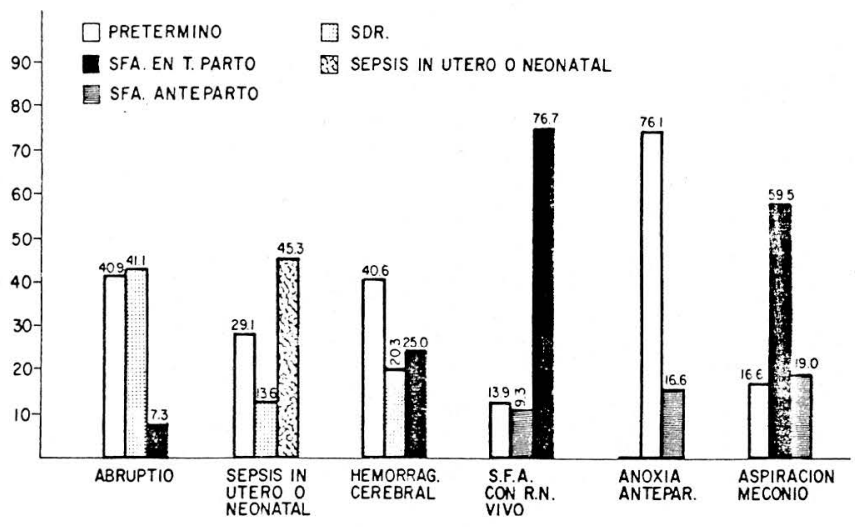

GRAFICA 29

DISTRIBUCION DE LAS AUTOPSIAS REALIZADAS SEGUN COMPONENTES DE LA MORTALIDAD PERINATAL

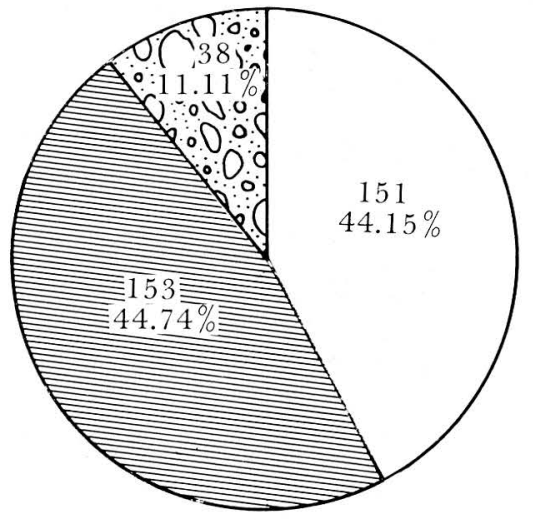

\section{INTRAPARTO}

ANTEPARTO

NEONATAL PRECOZ

SOBRE 1.823 CASOS INFORMADOS/175 SIN DATO

\subsection{Causas de la mortalidad perinatal según autopsia}

De las 342 autopsias realizadas, no se informó el resultado de la misma en 82 casos. De los $260 \mathrm{ca}-$ sos informados, más de la mitad, 168 (64.61\%) fueron fenómenos anóxicos, y en la tercera parte 83 (31.92\%), fueron anoxias anteparto en los cuales casi nunca se puede saber la etiología de la misma. Siguen en frecuencia las hemorragias $(11.92 \%)$, las infecciones $(8.08 \%)$, y las anomalías congénitas (7.39\%). (Gráfica No. 30).
GRAFICA 30

\section{CAUSAS DE LA MORTALIDAD PERINATAL SEGUN AUTOPSIA}

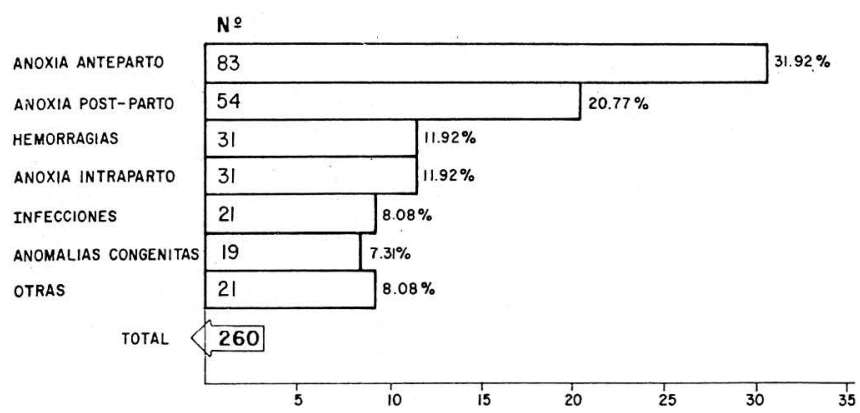

\subsection{Etiología de la anoxia anteparto según autopsia}

En la mayoría se desconoció la causa de la anoxia (68.68\%); sólo en la tercera parte de los casos de anoxia anteparto, $31.32 \%$, se conoció la etiología de la misma, atribuida en su mayoría al abruptio 16 casos (19.28\%). (Gráfica 31).

GRAFICA 31

\section{ETIOLOGIA DE LA ANOXIA ANTEPARTO SEGUN AUTOPSIA}

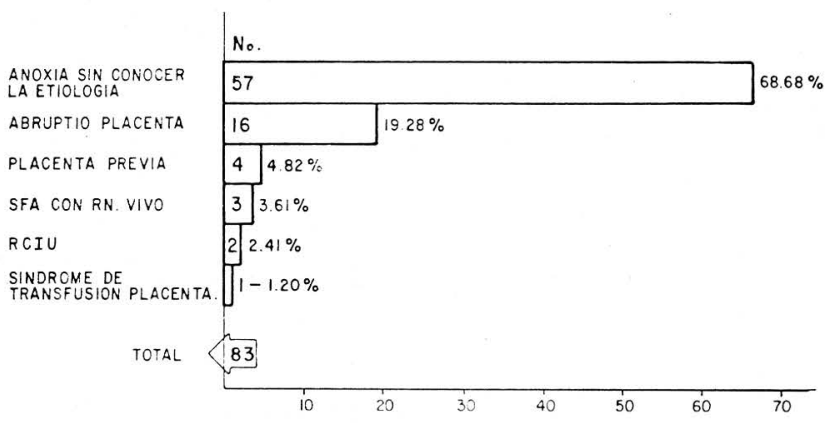

7.42. Etiología de la anoxia intraparto según autopsia

Más de la mitad de las anoxias intraparto (54.84\%) fueron debidas a alteraciones del cordón umbilical. En una tercera parte de las anoxias intraparto $35.48 \%$, se obtuvo un recién nacido vivo. (Gráfica 32).

\subsection{Etiología de la anoxia post-parto según autopsia}

En casi las tres cuartas partes de los casos se debió a la membrana hialina, $70.38 \%$, y casi una 
GRAFICA 32

ETIOLOGIA DE LA ANOXIA INTRAPARTO SEGUN AUTOPSIA

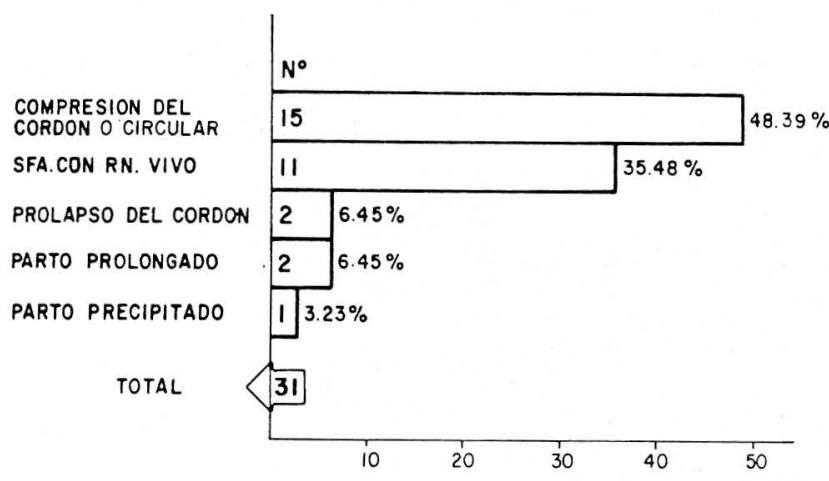

cuarta parte, $22.22 \%$, a la aspiración de meconio. (Gráfica 33).

GRAFICA 33

ETIOLOGIA DE LA ANOXIA POST-PARTO SEGUN AUTOPSIA

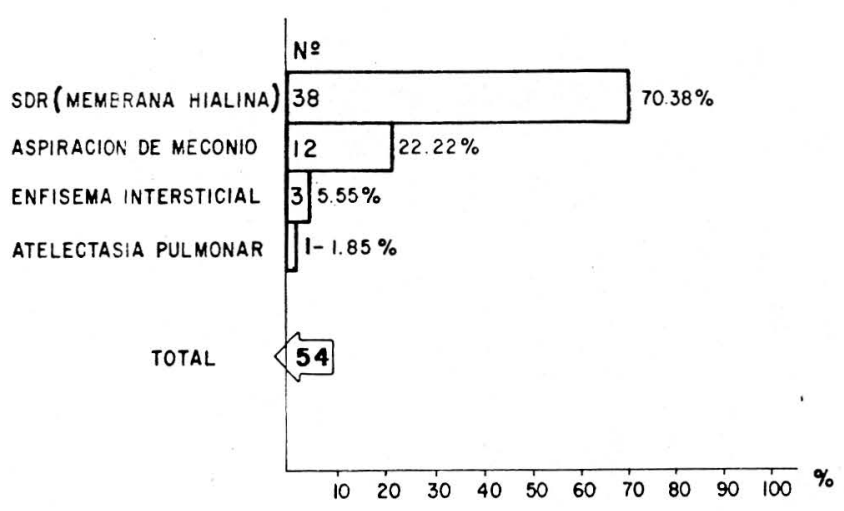

7.44. Etiología de la hemorragia del recién nacido según autopsia

Casi las tres cuartas partes de los casos, 22 (70.97\%), la etiología de la hemorragia del recién nacido fue cerebral. (Gráfica 34).

\subsection{Etiología de las infecciones del recién nacido según autopsia}

Un poco más de la mitad de los casos, $52.38 \%$, la etiología de la infección fue la sepsis in útero, que está de acuerdo con la corioamnionitis que ocupó en nuestro estudio el séptimo lugar dentro de las complicaciones del embarazo asociadas. (Gráfica 35).
GRAFICA 34

\section{ETIOLOGIA DE LA HEMORRAGIA DEL R.N. SEGUN AUTOPSIA}

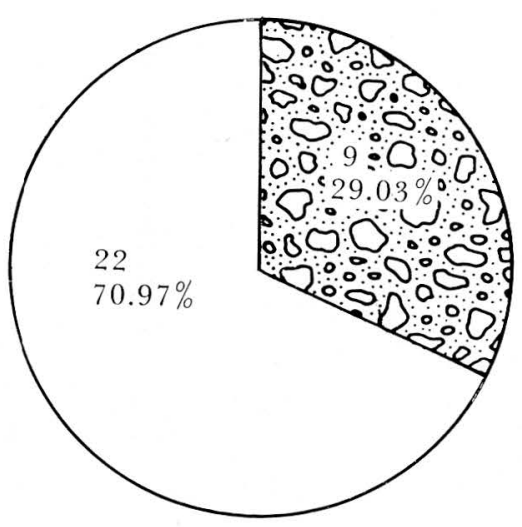

圆

HEMORRAGIA PULMONAR

HEMORRAGIA CEREBRAL

INFECCIONES DEL R.N. SEGUN AUTOPSIA

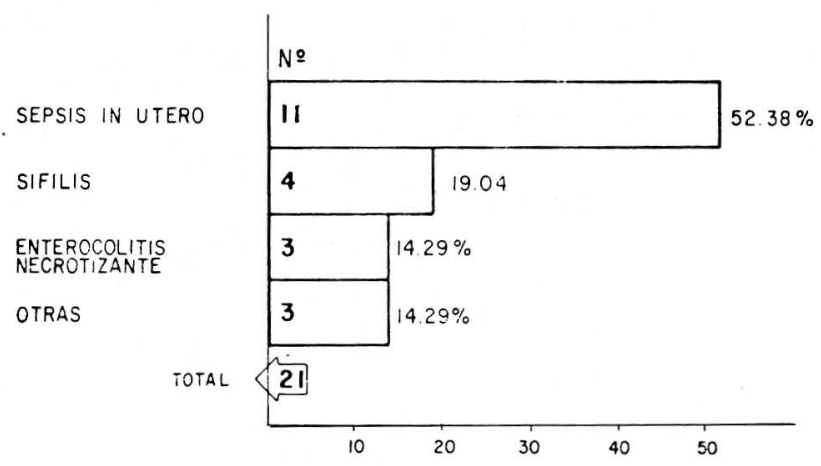

7.46. Anomalías congénitas según autopsia del recién nacido

La cuarta parte de las anomalías congénitas, $26.32 \%$, fueron meningomielocele. Las anomalías neurológicas constituyeron más de la mitad de todas las anomalías $52.63 \%$ y las anomalías cardiovasculares la tercera parte, 36.84\%. (Gráfica 36).

\subsection{Muertes perinatales prevenibles}

En el $55.14 \%$ de los casos la muerte fue clasificada como no prevenible, siendo un porcentaje importante $44.86 \%$, los casos catalogados como prevenibles. (Gráfica 37). 
GRAFICA 36

\section{ANOMALIAS CONGENITAS SEGUN AUTOPSIA}

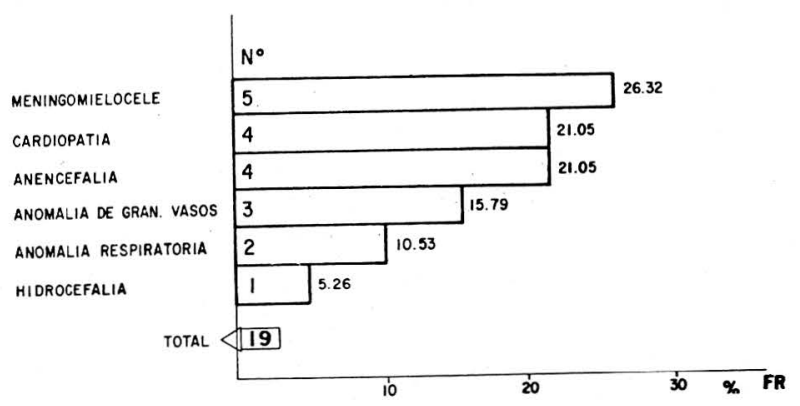

GRAFICA 37

MORTALIDAD PERINATAL PREVENIBLE

\section{NO}

\section{$55.14 \%$}

\section{DISCUSION DE RESULTADOS}

\subsection{Tasa de mortalidad perinatal según instituciones}

Comparando la tasa global con la encontrada en el estudio de Bogotá de 1985, observamos en casi todas las instituciones un aumento en la tasa de mortalidad perinatal. Como posible explicación de este incremento señalamos una mejoría en la calidad de la información como resultado de la creación de comités de mortalidad institucional.

Llama la atención la gran diferencia en las tasas de mortalidad entre hospitales de igual nivel de atención. En relación con este hallazgo es posible que la diferencia sea el resultado de atender un mayor número de pacientes de alto riesgo en una insti- tución que en otra; fenómeno que es más notorio en Bogotá, donde no en todos los hospitales de nivel III se cumple estrictamente con la regionalización.

Hay mayor número de instituciones de tercer nivel en nuestro estudio y ésto se debe a que la investigación ha sido realizada por la Federación Colombiana de Sociedades de Obstetricia y Ginecología (FECOLSOG), la cual tiene relación más directa con entidades universitarias que con las del estado. Si queremos aumentar la participación en este tipo de estudio de instituciones de segundo y primer nivel, es necesario que la división materno infantil del Ministerio de Salud se integre a la realización de estas investigaciones.

\subsection{Tasas de mortalidad comparativa entre Bogotá y el resto del país}

Entre las razones que podrían aducirse en la diferencia de tasas entre Bogotá y otras ciudades, para instituciones de tercer nivel, se encuentra que en Bogotá, hay hospitales de máxima tecnología que atienden casos de bajo riesgo en gran mayoría.

\subsection{Tasas de mortalidad perinatal según niveles de atención}

Creemos que la tasa de mortalidad, más elevada, encontrada para las instituciones del nivel III se debe a que en estos hospitales, se atiende un mayor número de pacientes de alto riesgo.

\subsection{Promedio de peso al nacer según componentes de la mortalidad perinatal}

Es notable que en todos los componentes de la mortalidad perinatal el promedio de peso de los recién nacidos es mayor de 2.000 gramos, peso suficiente para lograr supervivencia. Es muy importante encontrar un peso promedio de 2.725.50 gramos en el componente intraparto, hecho que cuestiona la calidad del control del trabajo de parto y parto.

El promedio de peso de los recién nacidos vivos que presenta muerte neonatal precoz es de 1.994.20 gramos, pero éste muy adecuado para lograr la supervivencia del recién nacido a menos que se hayan asociado factores patológicos diferentes al peso. 


\subsection{Distribución de la mortalidad perinatal según sus componentes}

El mayor porcentaje en el componente anteparto puede explicarse por ausencia o insuficiente control prenatal (42.\% de pacientes sin control prenatal). Si se efectúan acciones tendientes a mejorar la atención del parto y las unidades de cuidado neonatal, necesariamente el porcentaje de muertes perinatales intraparto y neonatal precoz debe disminuir.

\subsection{Complicaciones del parto asociadas con la mortalidad perinatal}

No siendo la cesárea una complicación del parto, sino un procedimiento, su segundo lugar no refleja necesariamente responsabilidad directa de la institución, sino el resultado de las causas que obligaron a realizar la cirugía. Igual aclaración es válida para el parto atendido con fórceps.

\subsection{Tipo de parto según componentes de la mortalidad perinatal}

Es de suponer que si la vigilancia y control del trabajo de parto es óptimo, la operación cesárea debe ser mayoría tanto en el componente intraparto como en el neonatal precoz, ya que es intervención que se realiza en muchos casos para resolver sufrimientos fetales intraparto y evitar en lo posible la muerte del producto durante el proceso del mismo.

En general, se observa que de acuerdo con el componente de la mortalidad perinatal, hay variaciones en el tipo de parto, toda vez que disminuye la proporción de parto vaginal y aumenta la de cesárea, siendo mayor la proporción de parto vaginal en el componente anteparto y la de la cesárea en el neonatal precoz. Esta observación concuerda con lo esperado.

\subsection{Autopsias realizadas}

El bajo porcentaje de autopsias es una limitante que no debe continuar presentándose en estudios de mortalidad que se realicen en Colombia. Por otra parte la autopsia del feto macerado aporta datos muy valiosos, aun siendo negativa. Entendemos que en la autopsia del macerado no es posible realizar el estudio microscópico. Pero los datos aportados por el estudio macroscópico son suficientemente importantes para justificarla. Las limitantes por costos y autorización de los padres son obstáculos a los cuales se les debe buscar solución. Los patólogos interesados en la mortalidad perinatal indican que el estudio de la placenta debe ser obligatorio junto con la autopsia del mortinato.

\subsection{Primeras causas de mortalidad perinatal según atención prenatal}

Es de anotar que el sindrome de dificultad respiratoria del R.N. y la anoxia anteparto, que ocupan la cuarta y quinta causa de muerte en el grupo con control prenatal, así como el desprendimiento prematuro de la placenta y la sepsis in útero o neonatal, que ocupan segundo y tercer lugar en el grupo sin control hacen suponer control prenatal de mala calidad, y la evidente falta del mismo respectivamente.

\subsection{Primeras causas de mortalidad perinatal según tipo de presentación}

Llama la atención que, en general, independiente del tipo de presentación la llamada anoxia anteparto, la cual a su vez involucra una serie de causas subyacentes no identificadas, es la principal participante en los casos de mortalidad perinatal; a su vez la membrana hialina, que es la segunda en prevalencia, hace pensar que la causa inherente a la prematuridad, es la causa más importante como factor de riesgo en la mortalidad perinatal en nuestro medio.

\subsection{Etiología de la anoxia post-parto según autopsia}

En lugares donde la atención perinatal es buena, causas como membrana hialina y aspiración de meconio, han disminuido el aporte de casos a la mortalidad perinatal, en forma importante; en cambio, en la presente investigación, estas causas siguen siendo prioritarias.

\subsection{Muertes perinatales prevenibles}

Es necesario aclarar que en algunas instituciones la definición de muerte prevenible o no, fue realizada con certeza esta calificación, cuando el número de autopsias es tan reducido.

\section{COMENTARIOS}

9.1. La creación de comités de mortalidad perinatal tiene un efecto positivo en las instituciones, permitiendo la detección oportuna de los casos, 
una mejor calidad en el registro y el análisis periódico de las causas de mortalidad perinatal, facilitando intervenciones que contribuyan a mejorar su situación de salud perinatal.

9.2. El sistema de regionalización a pesar de que está operando hace varios años, no logra. un funcionamiento óptimo bien sea por deficiencia en las condiciones mínimas de eficiencia que no permiten el funcionamiento esperado para cada nivel de atención, por subutilización del recurso físico y humano o por ausencia de un proceso normativo basado en el enfoque de riesgo que garantice la remisión oportuna y racional de la embarazada y el recién nacido.

9.3. Las dos terceras partes de las muertes perinátales ocurrieron durante el período fetal tardío, siendo el $82 \%$ de ellas anteparto y el $18 \%$ intraparto. Llama la atención el hecho de que las muertes anteparto se presentaron en su gran mayoría, en los hijos de gestantes que consultaron a las instituciones de salud con feto muerto, siendo imposible por el diseño de la investigación evaluar la proporción de esas pacientes que tuvieron control prenatal. Sin embargo, este hecho permite plantear una deficiencia marcada en el comportamiento de educación comunitaria de la salud perinatal, lo cual no favorece el reconocimiento por parte de la embarazada de la importancia de la capacitación precoz y el contacto periódico con instituciones de salud durante el embarazo, limitando al máximo el proceso de autocuidado y autogestión.

Otro factor que influye de manera importante es la pobre calidad del control prenatal que se realiza en la actualidad en algunas instituciones del país, observándose la tendencia del Miembro del equipo de salud a la evaluación de algunas variables generales y no al análisis adecuado de cada una de ellas, dentro del concepto de enfoque de riesgo.

9.4. La mortalidad fetal intraparto que representa el $12.36 \%$ del total de la mortalidad perinatal y el $18 \%$ de la mortalidad fetal tardía, es tres veces superior a lo esperado. Teniendo en cuenta que la mayoría de los partos ocurrieron en instituciones de nivel III de tipo universitario, llama la atención que en el $17.7 \%$ de las muertes perinatales el trabajo de parto duró más de doce horas y que la mortalidad intraparto está muy asociada a las presentaciones podálicas y distócicas, lo que plantea una deficiente calidad y supervisión en la atención del parto.
9.5. El $36.19 \%$ de las muertes perinatales ocurren durante el período neonatal precoz, siendo el promedio de peso de los recién nacidos que mueren antes del séptimo día de 1.994 gramos y el apgar al quinto minuto mayor de siete, en el $42.4 \%$ de las muertes neonatales; ésto plantea una ausencia de indicadores de riesgo sensible a la muerte neonatal precoz y una limitación en las condiciones de eficiencia, que no permiten el manejo adecuado del recién nacido de riesgo en los niveles II y III.

9.6. Las complicaciones hipertensivas del embarazo, la ruptura prematura de membranas y la amenaza del parto prematuro, son las patologías maternas más asociadas. El parto prematuro, la operación cesárea y las alteraciones de la dinámica uterina son las complicaciones del parto más asociadas. La prematurez y el sindrome de dificultad respiratoria del recién nacido son las complicaciones del neonato que en mayor proporción se asociaron con la mortalidad neonatal precoz. Es importante destacar que la amenaza del parto prematuro y la prematurez son las patologías asociadas más predominantes, al analizar cada uno de los componentes de la mortalidad perinatal.

9.7. Es necesario tener en cuenta que los diagnósticos de causa de defunción fueron asignados de acuerdo con criterios del comité en el $81.24 \%$ de las muertes perinatales; y en el $18.76 \%$ restante la causa fue asignada de acuerdo con el resultado de la autopsia.

En los casos de los cuales el comité asignó la causa de defunción, seguida por el sindrome de dificultad respiratoria del recién nacido, el desprendimiento prematuro de la placenta, la sepsis in útero o neonatal y la compresión del cordón umbilical.

En aquellos casos en los cuales se realizó la autopsia, la anoxia anteparto, la anoxia intraparto, la anoxia post-parto, las hemorragias y las infecciones fueron las causas más frecuentes. Al analizar la etiología de las diferentes anoxias se encontró que en el anteparto la anoxia de etiología desconocida era la causa más frecuente con el $68.68 \%$ de las muertes; en el intraparto, la compresión del cordón umbilical y el sufrimiento fetal agudo con el $83.87 \%$ de las muertes; y en el post parto la membrana hialina y la broncoaspiración de meconio con el $92.60 \%$ de las muertes. A partir de lo anterior se deduce que la anoxia anteparto como causa de muerte, es asignada con frecuencia en aquellos casos en los cuales se desconoce la real etiología de la muerte. Es importante insistir en la necesidad de practi- 
car un mayor número de autopsias y en la unificación de los diagnósticos de causas básicas de defunción un tanto para el componente fetal tardío, como para el componente neonatal precoz en todas las instituciones.

9.8. Llama poderosamente la atención, que cuando la causa de muerte neonatal precoz fue el sindrome de dificultad respiratoria del recién nacido, la alteración del parto más frecuentemente asociada fue la operación cesárea; observación hecha ya por otros investigadores y que amerita un estudio cuidadoso con otro tipo de diseño metodológico, teniendo en cuenta el incremento notorio que se ha encontrado en el país en la práctica de este procedimiento.

\section{CONCLUSIONES}

10.1. La tasa de mortalidad perinatal encontrada en el estudio de $23.8 \times 1.000$ es superior a la informada por los países industrializados.

10.2. La creación de comités de mortalidad perinatal permite el análisis de las causas de defunción, facilitando intervenciones que procuren la mejoría de la salud perinatal.

10.3. Las condiciones de eficiencia, para el manejo del recién nacido de riesgo en los niveles II y III no son óptimas.

10.4. Las complicaciones hipertensivas del embara zo, la ruptura prematura de membranas y la amenaza de parto prematuro, son las complicaciones maternas más asociadas.

10.5. El parto prematuro y las alteraciones de la dinámica del parto, son las complicaciones del parto más asociadas.

10.6. La prematurez y el sindrome de dificultad respiratoria del recién nacido, son las complicaciones del neonato más asociadas.

10.7. El número de autopsias realizadas es muy reducido.

10.8. Las cuatro primeras causas de muerte perinatal asignadas por los comités de mortalidad perinatal son en su orden:

- Muerte fetal por anoxia anteparto

- Sindrome de dificultad respiratoria
- Desprendimiento prematuro de la placenta

- Sepsis in útero o neonatal

Estas constituyen el $80 \%$ de todas las causas de mortalidad perinatal.

10.9. Las cuatro primeras causas de mortalidad peorden: rinatal diagnosticadas por autopsia son en su

- Anoxia sin conocer la etiología

- Abruptio placenta

- Compresión o circular de cordón

- Sindrome de dificultad respiratoria del R.N.

10.10. El diagnóstico de "Anoxia anteparto" en su gran mayoría refleja el desconocimiento de la causa real de la muerte.

10.11. Cuando la causa de la muerte neonatal fue el sindrome de dificultad respiratoria, la alteración del parto más frecuentemente asociada fue la operación cesárea.

10.12. Las causas más frecuentes de mortalidad perinatal encontradas en nuestro estudio pueden reducirse con la aplicación de medidas preventivas y terapéuticas disponibles en nuestro medio.

\section{RECOMENDACIONES}

11.1 Estimular la creación de comités permanentes de mortalidad perinatal en las instituciones de salud del país.

11.2. Diseñar un sistema de clasificación de causa básica de defunción para cada uno de los tres componentes de la mortalidad perinatal, que permita una asignación más real de la etiología de la muerte, modificando el instrumento vigente.

11.3. Implantar un sistema básico de registros a nivel nacional.

11.4. Diseñar e implementar a nivel nacional normas básicas para el control prenatal, atención del parto y manejo de las causas más frecuentes de mortalidad perinatal en nuestro medio.

11.5. Fortalecer el proceso de educación a la comunidad en salud perinatal.

11.6. Estimular procesos de investigación-acción en el país orientados a modificar la situación de la salud perinatal encontrada en el estudio. 
11.7. La FECOLSOG y la división materno-infantil del Ministerio de Salud Pública deben aunar esfuerzos con el fin de estimular los grupos perinatales a nivel local, regional y nacional que puedan conducir estudios relacionados con la mortalidad perinatal y materna en forma permanente.
Sólo conociendo nuestras verdaderas cifras de mortalidad durante el proceso reproductivo podríamos normatizar y corregir las causas que la están desencadenando.

\section{AGRADECIMIENTOS}

Al profesor Germán Ochoa M. por su valiosa orientación y asesoría.

A la profesora Yolanda Torres de Galvis, docente de Epidemiología de la Facultad Nacional de Salud Pública, por su constante colaboración para el análisis de los resultados.

$\mathrm{Al}$ doctor Augusto Hernández por el interés que mostró por el presente estudio.

Al doctor William Mejía por la realización de las pruebas estadísticas.

Al doctor Jorge Devia por su desinteresada asesoría en la programación y diagramación del informe final.

A Heriberto Henao C. estadígrafo y a John A. Muñetón por la colaboración en el procesamiento de la información y elaboración de las gráficas.

A todo el personal del Departamento de Obstetricia y Ginecología sin cuya participación directa o indirecta habría sido imposible concluir el presente estudio.

Al señor Alberto Chica de la Imprenta de la Universidad de Antioquia por el interés que brindó para la publicación del presente trabajo.

\section{BIBLIOGRAFIA}

1. ABUDU, O; AKINKUBE A. "Clinical causes and classification of perinatal mortality in lagos". Int. J. Gynecol Obstet. 1982 Dec; 20(6): 443-7

2. BARNS, T. "Obstetrics in the third world with particular reference to field research into delivery of maternal care to the community'. En: Bourne C, en: Recent advances in obstetrics and Gynecology. Edimburgh: London, 1979; Churchill. Livingstone.

3. BOWES, WA Jr. "A review of perinatal mortality in Colorado, 1971 to 1978 , and, its relationship to the regionalization of perinatal services'. Am. J. Obstet Gynecol 1981 Dec. 141 (8): 1045-52.

4. BOYLAN, P.O.; DRISCOLL, K. "Improvement in perinatal mortality rate attributed to spontaneous preterm labor without use tocolytic agents". Am. J. Obstet Gynecol 1983 Apr 1; 145(7): 781-3.
5. DAIKOKU, NH; KALTREIDER, DF; JOHNSON, TR Jr; JOHNSON, JW; SIMNONS, MA. "Premature rupture of membranes and preterm labor: Infection and perinatal mortality risks"'. Am. J. Public Health 1981 Jan; $71(1)$ : 38-46.

6. DIAZ, R. "Mortalidad perinatal en Bogotá, 1983 1984 Secretaría de Salud del Distrito Especial de Bogotá, Colombia, cifras oficiales de certificados de defunción y de natalidad". En proceso de publicación.

7. ELIZAGA, JUAN C. "Métodos Demográficos para el estudio de la mortalidad". Centro Latino Americano de Demografía, 1969, Serie E, No. 4.

8. ERKOLA, R.; KERO, P.; SEPP, "AL" A.; GR'ONROOS, M.; RAURAMO, L. "Monitoring perinatal Mortality by birth weight specific Mortality rates". Int J. Gynecol. Obstet. 1982. Jun; 20(3); 231-5. 
9. GAVIRIA, B. et. al. "Lealtad Perinatal" Revista Colombiana de Obstetricia y Ginecología 11(3): 389$50,1960$.

10. HOSKINS, EM.; ELLIOT, E.; SHENNAN, AT.; SKINDMORE, MB.; KEITH, E. "Out come of very low-birth weight infants born at a Perinatal Center" Am. J. Obstet. Gynecol. 1983. Jan 15; 145(2): 13540.

11. INSULL, B.J.; MAC LEAN, NE.; BLANCHETTE, G. "Perinatal Mortality in Southland". NZ med J. 1980 Jan 23; 91 (652): 40-2.

12. JUBIZ, A.; LONDOÑO, J. "Morbilidad Perinatal" Revista Col. de Obstetricia y Ginecología 35(6): 440-64, 1984.

13. KASS, E.H. "infectious disease and perinatal morbidity”. Yale J. Biol. med. 1982. May-Aug; 55(3-4) $231-7$.

14. KNUTZEN, VK.; SHER, G. "The major determinants of perinatal mortality in a large metropolitan Hospital. Results of a retrospective study". J Reprod. med. $1982 \mathrm{Jul} ; 27(7) ; 395-400$.

15. LANGER, A.; ARROYO, P. "La mortalidad perinatal en el Instituto Nacional de Perinatología, México". Bol.Med.Hosp., Infant. Mex. Vol. 40,número 11,Nov. 1983.

16. LEE, K.; TSENG, P.; EIDELMAN, A.; KANDALL, S.; GARTNER, L. "Determinants of the neonatal mortality”. Am. J. Dis. Child 1976; 130:842.

17. LINDGREN, L. "Perinatal mortality and different socieconomic status”. Acta Obstet. Gynecol. Scand $1981 ; 60(1): 21-6$.

18. LOPEZ, G.; RIAÑO, G. "Atención del Parto Hospitalario en Colombia: Primeros hallazgos" Monografías de la CCRP; Vol. 11; Junio 1979.

19. NAEYE, RL. "Abruptio Placentae and Placenta previa: Frecuency, perinatal mortality, and cigarette smoking". Obstet. Gynecol. 1980 Jun; 55(6) 701-4.

20. OCHOA, G. "Mortalidad perinatal" Antioquia Médica 8(1): 2-53, 1958.

21. OCHOA, G. et. al. "Resultado perinatal" Escuela Nacional de salud pública y facultad de medicina, Universidad de Antioquia. OMS/OPS. Informe preliminar.

22. O' DRISCOLL, K.; FOLEY, M. "Correlation of decrease in perinatal mortality and increase in cesarean section rates'. Obstet. Gynecol. 1983 Jan; 6(1) 1-5.
23. OMS "La prevención de la mortalidad perinatales" (Informe de un seminario) cuadernos de salud pública No. 42 Ginebra. 1972.

24. OPS - OMS. "Clasificación Internacional de enfermedades”. Vol. 1 - 1975.

25. OPS "Manual sobre enfoque de riesgo en la atención materno infantil" 1986 . No. 7 .

26. PAEZ, R.; ACERO, U.; URIBE, H. "Mortalidad Perinatal" Rev. Col. de Obst. y Ginecología. Vol. XXV No. 5 - 349-58, 1974.

27. PEARSON, JW. "Cesarean section and perinatal mortality. A nine year experience in a city country Hospital" Am. J. Obstet. Gynecol. 1984 Jan 15; 148(2), 155-9.

28. PERKINS, RP. "The neonatal significance of selected perinatal events. Among infants of low birth weight II The influence of ruptured membranes". Am. J. Obstet. Gynecol. 1982 Jan 1; 142(1): 7-16.

29. RODRIGUEZ, ARTURO; MUNOZ, LUIS ARMANDO; TAMARA, ALFREDO; LAGOS, MANUEL. "Mortalidad Perinatal: Estudio de muestreo Instituto Materno Infantil de Bogotá”.

30. SALUD PERINATAL. "Boletín del Centro Latinoamericano de Perinatología y Desarrollo Humano" Vol. 2 No. 4 de 1985.

31. SALUD PERINATAL. "Boletín del Centro Latinoamericano de Perinatología y Desarrollo Humano". Vol. 1 No. 3 de 1984.

32. STUBLEFIELD, PG.; BEREK, JS. "Perinatal mortality in term and post-term births". Obstet. Gynecol. 1980 Dec; 56(6) 676-82.

33. TERRIN, M.; MEYER, MB. "Birth weight specific rates as a bias in the effects of smoking and other perinatal hazzards”. Obstetric Gynecol. 1981 Nov. 58(5) 636-8.

34. URIZA-BARRAGAN Y COLMENARES. "Mortalidad perinatal. Estudio cooperativo institucional". Rev. Col. de Obst. y Ginec. Marzo-Abril 1986, Vol. XXXVII No. 2 Pág. 91-107.

35. WIGGLESWORTH, JONATHAN S., M.D. "Perinatal Pathology" Vol. 15 is the series 1984 by W.B. Sannders Company. MPPLS. 